\title{
UV- and Midwave-Sensitive Cone-Driven Retinal Responses of the Mouse: A Possible Phenotype for Coexpression of Cone Photopigments
}

\author{
A. L. Lyubarsky,, ${ }^{1,2}$ B. Falsini, ${ }^{3}$ M. E. Pennesi, ${ }^{1,2}$ P. Valentini, ${ }^{3}$ and E. N. Pugh Jr' ${ }^{1,2}$ \\ ${ }^{1}$ Department of Psychology and ${ }^{2}$ Institute of Neurological Sciences, University of Pennsylvania, Philadelphia, \\ Pennsylvania 19104-6196, and 3/nstitute of Ophthalmology, Catholic University, Rome, Itay 00168
}

\begin{abstract}
Molecular biological, histological and flicker electroretinographic results have established that mice have two cone photopigments, one peaking near $350 \mathrm{~nm}$ (UV-cone pigment) and a second near $510 \mathrm{~nm}$ [midwave (M)-cone pigment]. The goal of this investigation was to measure the action spectra and absolute sensitivities of the UV-cone- and M-cone-driven $\mathrm{b}$-wave responses of C57BL/6 mice. To achieve this goal, we suppressed rod-driven signals with steady or flashed backgrounds and obtained intensity-response relations for conedriven b-waves elicited by narrowband flashes between 340 and $600 \mathrm{~nm}$. The derived cone action spectra can be described as retinal ${ }_{1}$ pigments with peaks at 355 and $508 \mathrm{~nm}$. The UV peak had an absolute sensitivity of $\sim 8 \mathrm{nV} /\left(\right.$ photon $\left.\mu \mathrm{m}^{2}\right)$ at the cornea, approximately fourfold higher than the $\mathrm{M}$ peak. In an attempt to isolate UV-cone-driven responses, it was discovered that an orange conditioning flash $(\lambda>530 \mathrm{~nm})$ completely
\end{abstract}

The mouse has become an increasingly important preparation for the investigation of retinal function and retinal disease. This importance comes from general use of the mouse as a model system for mammalian gene mapping and manipulation, and from the specific development of lines of mice having transgenes for retinally expressed proteins (Lem and Makino, 1996). Because rodents such as the mouse have traditionally been characterized as having "rod-dominated" retinas, and because signals originating in cone photoreceptors are so important in human vision, concern has been expressed that the mouse may be an inadequate model system for investigation of the retinal processing of cone signals. That mice have cones is certainly incontrovertible.

Carter-Dawson and LaVail (1979a,b) performed a definitive histological assessment of the photoreceptor types of the mouse retina and estimated that cones constitute $3-3.5 \%$ of the total number of photoreceptors. Subsequent studies using flicker ERG photometry under cone-isolation conditions found that the mouse retina has two distinct spectral peaks of sensitivity, one at 360$365 \mathrm{~nm}$ and a second at $511 \mathrm{~nm}$ (Jacobs et al., 1991; Deegan and Jacobs, 1993). Experiments with regional flicker stimulation later confirmed the UV sensitivity to be higher in ventral retina and

\footnotetext{
Received July 13, 1998; revised Oct. 19, 1998; accepted Oct. 21, 1998.

This work was supported by National Institutes of Health Grant EY-02660, and the Penn Therapeutic Initiative for Retinitis Pigmentosa. B.F. was supported by a fellowship from the Fulbright Foundation.

Correspondence should be addressed to Dr. E. N. Pugh Jr, University of Pennsylvania, Department of Psychology, 3815 Walnut Street, Philadelphia, PA 191046196.

Copyright (ㄷ) 1998 Society for Neuroscience $\quad 0270-6474 / 98 / 190442-14 \$ 05.00 / 0$
}

suppressed ERG signals driven by both $M$ pigment- and UV pigment-containing cones. Analysis showed that the orange flash could not have produced a detectable response in the UV-cone pathway were their no linkage between $M$ pigmentand UV pigment-generated signals. Because cones containing predominantly the UV and M pigments have been shown to be located largely in separate parts of the mouse retina (Szel et al., 1992), the most probable linkage is coexpression of $M$ pigment in cones primarily expressing UV pigment. New histological evidence supports this interpretation (Gloesman and Ahnelt, 1998). Our data are consistent with an upper bound of $\sim 3 \%$ coexpression of $\mathrm{M}$ pigment in the cones that express mostly the UV pigment.

Key words: cone photoreceptors; cones; cone pigments; murine retina; retinal sensitivity; electroretinogram; retinal circuitry; gene coexpression responsiveness to green light to be localized mostly in its dorsal part (Calderone and Jacobs, 1995).

Immunocytochemical studies also provided evidence of two distinguishable cone types in the murine retina (Szel et al., 1992). One type was labeled by a monoclonal antibody specific to the midwave- to long-wave-sensitive visual pigment of the mammals, whereas the other type was stained by a short-wave (S) conespecific monoclonal antibody. The same study provided the first evidence that the midwave-sensitive cones are located exclusively in the dorsal half of the mouse retina, whereas the overwhelming majority of the short-wave-sensitive cones occupy the ventral half (Szel et al., 1992).

Molecular biological screening has also confirmed the existence and expression of two distinct cone pigments in mouse, and sequence and spectral analyses have shown one of these to be a member of the "LW" cone photopigment family [of which the human L-and midwave (M) cone pigments are members] and the second to be a member of the "S" cone photopigment family (of which the human S-cone pigment is a member) (Chiu et al., 1994; Sun et al., 1997). The S-cone family contains members whose peak sensitivity ranges from 350 to $450 \mathrm{~nm}$ or higher (Chiu et al., 1994).

In summary, three bodies of evidence-electroretinographic, histochemical, and molecular biological-have converged in showing the mouse to have cones of two distinct classes and thus in providing support for use of the mouse as a model for the investigation of signals originating in cones. We undertook this investigation to isolate ERG responses driven by murine UV- and midwave-sensitive cones, and to characterize more fully than 
before the absolute flash sensitivities, the spectral sensitivities, and the magnitudes and kinetics of these responses.

\section{MATERIALS AND METHODS}

Animal maintenance and light regimen. All experimental procedures were done in compliance with National Institutes of Health guidelines as approved by the University of Pennsylvania's Institutional Animal Care and Use Committee. Animals used in the study were born and maintained under strictly controlled ambient illumination throughout their lives. Breeding pairs were set in light-tight, ventilated enclosures lit from inside by four $25 \mathrm{~W}$ tungsten lamps situated behind a diff using screen; the lamps were powered by a regulated power supply and controlled by a timer. The interior of the containers was painted with a flat-white epoxy paint, with the result that interior illumination was practically isotropic. Animals were maintained on a $12 \mathrm{hr}$ light/dark cycle, with the illumination level at 2.5 photopic lux $(\approx 7.5$ scotopic lux). In a ganzfeld such illumination has been estimated to produce approximately 650 photoisomerizations/sec in the rods of an adult mouse with a fully dilated pupil, but no more than 100 photoisomerizations/sec in the rods of an adult mouse with the pupil fully constricted (Pennesi et al., 1998). Servicing of the mice was performed three times a week, exposing them to 7-10 lux illumination for 20-25 min. These precautions about illumination rearing conditions were taken to obviate any potential retinal damage from stronger light exposures and also in an effort to minimize variability between animals.

$E R G$ recording. On the day preceding the experiment animals were removed from the containers and placed into a completely darkened light-tight ventilated enclosure. By the time of the experiment the animals had spent $12-20 \mathrm{hr}$ in dark. All of the preparations for recordings were performed under dim red light. The animals were anesthetized with an intraperitoneal injection of a solution containing (in $\mu \mathrm{g} / \mathrm{g}$ body weight): 25 ketamine, 10 xylazine, and 1000 urethane. The average length of time under anesthesia was $1.5 \mathrm{hr}$, with a range of $1-2 \mathrm{hr}$. The pupil was dilated with $1 \%$ tropicamide saline solution (Mydriacil; Alconox, New York, NY), and the animal was immobilized in a holder. A drop of methylcellulose solution (Goniosol; Iolab Pharmaceutical, Indianapolis, IN) was placed on the eye for protection and electrical contact, and a recording platinum wire electrode was put into electrical contact with the cornea while a needle tungsten reference electrode was inserted subcutaneously on the forehead. After this the animal was placed into a light-proof Faraday cage and kept in absolute darkness for 10 min before recordings commenced. The temperature inside the cage was maintained at $27 \pm 1^{\circ} \mathrm{C}$. Signals were amplified by a BMA-200 differential amplifier (GWE Inc., Ardmore, PA), bandpass-filtered at 0.1-1000 Hz (two-pole Butterworth filter), sampled at $5 \mathrm{kHz}$, and stored on the hard drive of a personal computer using Digidata 1200 acquisition hardware and Axotape 2 software (Axon Instruments, Foster City, CA).

Light stimulation. The Faraday cage in which the recordings were made was an 8 inch $\times 8$ inch $\times 14$ inch aluminum chamber whose interior was completely covered with aluminum foil, serving to create a ganzfeld (described further below). The use of aluminum foil as an interior cover was dictated by the need to minimize the UV absorbance of the chamber walls, which we found to occur to an unacceptable degree with commercially available paints. A custom animal holder and support allowed the mouse to be placed at almost any location inside the chamber. Thus, the ERGs of either eye could be recorded, though in the majority of experiments only the right eye was used. In all of the experiments reported here the holder was adjusted to keep the optic axis of the eye from which the ERG was being recorded vertical, at a height about two-thirds above the chamber floor and in the center of its horizontal cross section.

Light stimuli were delivered through several ports in the walls and ceiling of the box. Special care was taken to avoid any possibility of direct illumination of the tested eye from any of these openings. To achieve this the ceiling ports were protected by foil-wrapped baffles of appropriate size, and the wall ports were located at the bottom of the chamber, much lower than the animal holder, which served as a light baffle.

The directional homogeneity of the light field at the location of the mouse's pupil was checked as follows. A PIN-10 photodiode (UDT, Waltham, MA) with a custom-made accessory that limited light collection to an angular subtense of $\sim 2^{\circ}$ [for an explanatory drawing, see Lyubarsky and Pugh (1996), their Fig. 1] was placed at the position occupied by the mouse eye during experiments, and the intensity of light from different directions was measured. For an angular subtense of $120^{\circ}$ about the mouse's optic axis, variations of light intensity did not exceed $\pm 15 \%$ of the average intensity, regardless of the port used to deliver stimulation.

The optical stimulator allowed considerable freedom in the choice of light sources and protocols of stimulation. In this study we used three independently powered and triggered flash illuminators built around U-8538 xenon flash tubes (Mouser Electronics, Randolph, NJ) and one steady light illuminator. The flash illuminators were powered by switchable capacitor banks (up to $1000 \mu \mathrm{F}$ ) charged to $450 \mathrm{~V}$, and they provided up to 100 joules/flash to the lamp. These flashlamps discharged $>85 \%$ of their light output in $1 \mathrm{msec}$. The steady illuminator used a $12 \mathrm{~V} / 50 \mathrm{~W}$ halogen lamp (HLX 64610, Osram, Berlin, Germany) powered by a stabilized DC supply; steady illumination was under the control of a shutter (Uniblitz; Vincent Associates, Rochester, NY). The illuminators delivered their light directly to the appropriate ports. The intensity and spectral composition of illumination were controlled by quartz neutral density, color glass, and bandwidth interference filters (Ealing Electrooptics, Holliston, MA) introduced into appropriate places in the beams. The transmission spectra of all filters over the spectral range $300-700 \mathrm{~nm}$ were measured with a Lambda 20 spectrophotometer (Perkin-Elmer, Norwalk, CT). Flashes were triggered by a computer with a digital input/output board (Computer Boards, Mansfield, MA).

Overview of light calibrations, filter spectral transmission measurements, and estimation of photoisomerization numbers and rates in rods and cones. Although light calibration is normally straightforward, some requirements of these experiments called for unexpectedly difficult calibration measures. One such requirement was that of working with UV stimuli in a ganzfeld. Another was the need to maximize stimulation intensity in the recording chamber while keeping stimuli as narrowband as possible. The latter requirement led us to use noncollimated beams, which broadened and shifted the interference filter transmission characteristics from the manufacturer's specifications and forced us to measure the transmission characteristics in a flash beam geometry matching that used in the experiments. Finally, because of the goal of characterizing the sensitivity of cone-driven responses in absolute and physiologically meaningful units, we have laid out in detail the steps used to estimate the numbers and rates of photoisomerizations in rods and cones produced by the light stimuli. The reader who is not interested in such detail may prefer to skip to the final section of Materials and Methods.

Measurement and quantification of light stimuli: basic method. Light from each of the optical stimulators was calibrated as follows. A factorycalibrated silicon photodiode (PIN-10 or PIN-5DP/DB, UDT) was placed with working surface up at the position normally occupied by the mouse's eye during an experiment. Narrowband interference filters (full width, half-maximum transmission $<9 \mathrm{~nm}$ ) with transmission maxima ranging from 350 to 600 were positioned in a beam near the source, and the photodiode current in response to a flash or step of light was measured with a current-to-voltage transducer, with neutral density filters interposed in the beam as needed to keep the photodiode current below $10 \%$ of its saturating magnitude. The transducer's output signal was sampled at $200 \mathrm{kHz}$, and the photon flux density at the pupil plane for light flashes was computed as follows (Wyszecki and Stiles, 1983):

$$
Q\left(\lambda_{\text {nom }}\right)=\frac{\lambda_{\text {nom }} 10^{-9} 10^{D} \int i d t}{h c A_{\mathrm{pd}} s\left(\lambda_{\text {nom }}\right)} \text { photons } \mu \mathrm{m}^{-2} .
$$

Here $\lambda_{\text {nom }}(\mathrm{nm})$ is the manufacturer-specified (nominal) wavelength of the interference filter, $D$ is the optical density of any neutral density filters interposed in the beam, $Q\left(\lambda_{\text {nom }}\right)$ is the computed maximal (i.e., for $D=0$ ) photon density; $i$ (amps) the photodiode current, $A_{\mathrm{pd}}\left(\mu \mathrm{m}^{2}\right)$ the photodiode surface area, $s(\lambda)$ (amps watt ${ }^{-1}$ ) the photodiode sensitivity at wavelength $\lambda, h=6.63 \times 10^{-34}$ (joules $\mathrm{sec}^{-1}$ ) Planck's constant, $c=$ $3 \times 10^{17} \mathrm{~nm} \mathrm{sec}^{-1}$ the speed of light. For light steps, the steady current $i$ was substituted for the integral over time in Equation 1. The photodiode current $i$ in Equation 1 is induced by photons whose wavelengths vary over the band of the filter, and thus an integration over the passband occurs as the photodiode generates current; the integration over $t$ is performed explicitly (numerically) offline. Use of Equation 1 embodies the assumption that the finite bandwidth of the interference filters has negligible effect on the estimated total quantal flux $Q\left(\lambda_{\text {nom }}\right)$; this assumption is warranted because $s(\lambda)$ varies $<10 \%$ over the band of any specific filter. However, explicitly mentioning this assumption also raises an issue that turned out to be critical in the rod and cone spectral sensitivity measurements, namely, the wavelength $\lambda_{\text {nom }}$ to which the measured quantal flux is assigned during the analysis of spectral sensitivity data. This problem was complicated by the placement of the interference filters in the flash illuminator beams, as we now describe. 


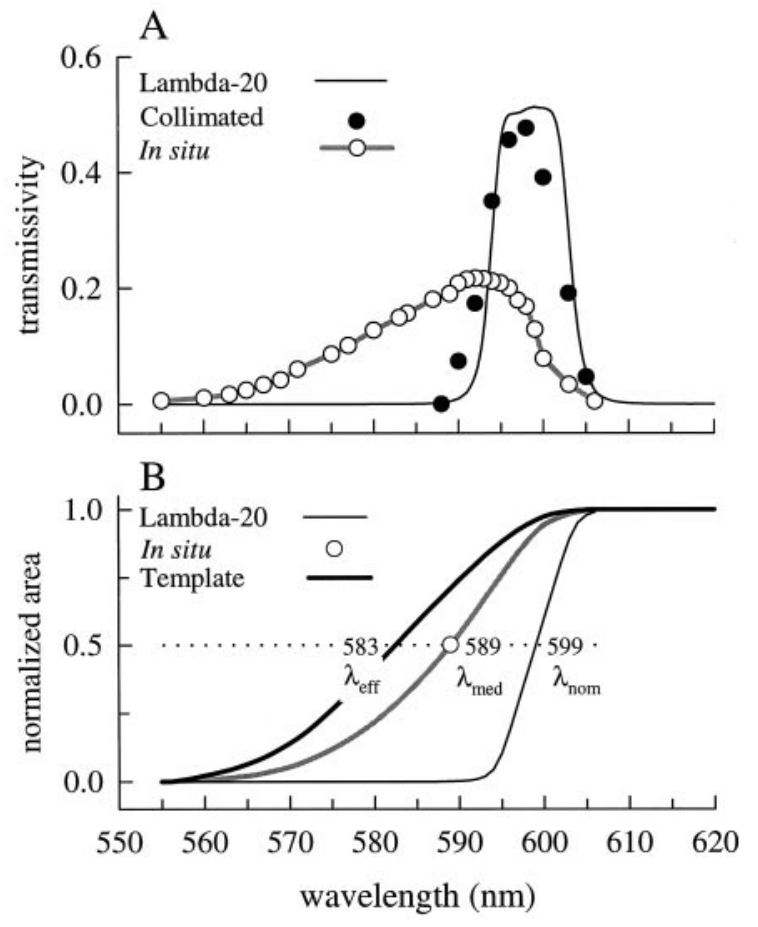

Figure 1. Effect of uncollimated sources on an interference filter transmission spectrum. A, The thin unbroken line presents the transmission spectrum of a nominal $600 \mathrm{~nm}$ interference filter recorded with a PerkinElmer Lambda-20 spectrophotometer. The open circles are the transmissivity values measured in situ (i.e., with the flash geometry used in the ERG investigation), obtained as described in the text; the filled circles give the transmissivities of the same filter measured with the same photodiode used to obtain the open circles, but with a collimated light beam. $B$, The thin unbroken line presents the integral of the transmission spectrum determined with the Lambda-20 spectrophotometer; it reaches 0.5 of its area at $599 \mathrm{~nm}$. The thickened gray line is the integral of the transmission spectrum measured in situ, in the uncollimated beam geometry of the experiments; the 0.5 point $(\bigcirc)$ is at $589 \mathrm{~nm}$. The thickened black line presents the integral used to determined $\lambda_{\text {eff }}$, which is seen to be $583 \mathrm{~nm}$ for the theoretically calculated in situ absorption spectrum of rhodopsin (see Fig. 6A; Eq. 8).

Measurement of narrowband interference filter transmission spectra. To achieve adequate intensities for cone-driven responses we found it necessary to use flash tubes whose size made it impossible to collimate their output into parallel light beams. The lack of beam collimation caused the effective spectral characteristics of the interference filters to differ significantly from specifications. Therefore, the transmission spectra of all interference filters had to be measured in an optical geometry identical to that used in the ERG experiments. Specifically, the filter whose transmission spectrum was to be measured was placed in the same holder attached to the same flash unit used in the experiments to deliver light into the recording chamber. For the spectral transmission determination, however, the light transmitted through the filter was delivered not to the recording chamber but rather to one port of an 8-inch-diameter integrating sphere (Ealing Electrooptics); the light exiting the second port of the integrating sphere was then passed through a concave grating monochromator having $1 \mathrm{~nm}$ bandwidth (H-20V, J-Y Optical Systems, Metuchen, NJ). Light intensity was then measured (as described above) at $1 \mathrm{~nm}$ steps with a photodiode attached to the exit slit of the monochromator. The transmission spectra of the filters were also obtained with the same monochromator in a configuration generating a collimated light beam through the filter, for comparison with the manufacturer's specifications. Figure $1 A(\bigcirc)$ presents transmission spectra obtained as just described of a filter with manufacturer-specified maximum transmission at $\lambda_{\text {nom }}=600$ $\mathrm{nm}$. It is clear from this figure that the transmission spectrum obtained with the geometry used in the experiments (In situ) is both shifted to shorter wavelengths and broadened by the lack of beam collimation. As shown in Figure $1 B$, the shift was quantified by determining the wavelength $\lambda_{\text {med }}$ corresponding to median of the spectral transmission func- tion: in this instance, $\lambda_{\text {med }}$ was shifted from the value $599 \mathrm{~nm}$ obtained with the spectrophotometer measurement to $589 \mathrm{~nm}$ by the lack of beam collimation. Shifts of the same direction and ranging in magnitude from 3 to $10 \mathrm{~nm}$ were found for all of the interference filters.

Because of the finite bandwidth of the filters, and the effect of placement in the flash beam on the spectral transmission function, we must distinguish between the nominal wavelength (manufacturer's specification) of a filter and the wavelength assigned to the flash energy delivered in situ. In the immediately following sections in Materials and Methods, we will honor this distinction by subscripting the $\lambda$ when the distinction is material. In particular, throughout this manuscript, we adopt the convention of referring to the nominal (or manufacturer-specified) wavelength of a bandbass filter by $\lambda_{\text {nom }}$. When not subscripted, the $\lambda$ will refer to the wavelength of an ideal, monochromatic light.

Measurement of spectral densities of white flashes and flashes filtered by broadband color glass filters. In some experiments, to generate flashes of sufficiently high intensity we had to use "white" flashes, or flashes filtered with broad bandwidth color glass filters. To determine the spectral content of such flashes, we first estimated the emission spectrum of the unfiltered flash source at $10 \mathrm{~nm}$ spacing across the spectrum by making measurements with the narrowband interference filters and applying the formula:

$$
E_{\text {source }}\left(\lambda_{\text {med }}\right)=\frac{Q\left(\lambda_{\text {med }}\right)}{\infty} .
$$

Here $Q\left(\lambda_{\text {med }}\right)$ is the measured value obtained with Equation 1 (with $\lambda_{\text {med }}$ substituted for the nominal wavelength $\left.\lambda_{\text {nom }}\right), T_{\lambda_{m e d}}(\lambda)$ is the transmission spectrum of the narrowband filter determined in situ as described above, and $E_{\text {source }}\left(\lambda_{\text {med }}\right)$ is the emission (in photons $\mu \mathrm{m}^{-2}$ at the cornea) at the wavelength $\lambda_{\text {med }}$. The emission $E(\lambda)$ at all points in the spectrum was then computed by linear interpolation between the discrete measurements.

The efficacy of white flashes or flashes filtered by a broadband filter having spectrophotometrically measured transmission spectrum $T_{\mathrm{BB}}(\lambda)$ was calculated in terms of "equivalent photons at the pigment $\lambda_{\max }$ " with the formula:

$$
Q_{\text {equiv }}\left(\lambda_{\text {max }}\right)=\int_{0}^{\infty} E_{\text {source }}(\lambda) T_{\mathrm{BB}}(\lambda) \bar{S}_{\text {theory }}(\lambda) \mathrm{d} \lambda .
$$

Here $\bar{S}_{\text {theory }}(\lambda)$ is an expression for a normalized pigment spectrum, described below (Eq. 8).

Conversion of flash stimuli to numbers of photoisomerizations in rods and cones. Several factors must be taken into consideration to estimate from the quantal flux at the cornea the retinal flux density, and from the retinal flux density the number $(\Phi)$ or rate $(\varphi)$ of photoisomerizations per photoreceptor produced by a light flash or step. For a truly monochromatic flash delivering quantal flux density $Q(\lambda)$ (photons $\mu \mathrm{m}^{-2}$ at the cornea) in a ganzfeld

$$
\Phi=Q(\lambda) \tau(\lambda) \frac{A_{\text {pupil }}}{A_{\text {retina }}} a_{c}(\lambda),
$$

where $\tau(\lambda)$ is the transmissivity of the preretinal eye media at $\lambda$ (described below), $A_{\text {pupil }}, A_{\text {retina }}$ the areas of the mouse pupil and retina, and $a_{\mathrm{C}}(\lambda)$ the end-on collecting area at the retina of the specific photoreceptor type. The latter can be expressed (Baylor et al., 1984) as:

$$
a_{c}(\lambda)=f \frac{\pi d^{2}}{4}\left[1-10^{-\Delta D(\lambda) L}\right] \gamma
$$

where $d$ is the outer segment diameter, $\Delta D(\lambda)$ is the specific axial density of the visual pigment [optical density (o.d.) units $\mu \mathrm{m}^{-1}$ ], $L(\mu \mathrm{m})$ the outer segment length, $f \geq 1$ a dimensionless factor that accounts for any light funneling by the inner segment, and $\gamma$ the quantum efficiency of photoisomerization. Estimates of $a_{\mathrm{C}}\left(\lambda_{\max }\right)$ for mammalian rods computed with Equation 5 range from 0.9 to $2.3 \mu \mathrm{m}^{2}$ (Breton et al., 1994; Schneeweis and Schnapf, 1995; Lyubarsky and Pugh, 1996); we previously adopted the value $1.3 \mu \mathrm{m}^{2}$ for murine rods and will assume the latter value here.

For simplicity one can collapse all of the eye-dependent terms of Equation 4 into a single parameter; thus:

$$
\Phi=Q(\lambda) a_{\mathrm{C}, \text { cornea }}(\lambda)
$$


where $a_{\mathrm{C}, \text { cornea }}$ is the apparent collecting area of the specific photoreceptor type at the cornea in a ganzfeld. Previously, we estimated $a_{\mathrm{C}, \text { cornea }}$ for mouse rods to be $0.2 \mu \mathrm{m}^{2}$ at $\lambda=500 \mathrm{~nm}$ (Lyubarsky and Pugh, 1996), and we will adopt that value here. When the spectral density $Q(\lambda)$ is not monochromatic, photons of different wavelengths are summed in the photoreceptor, and so Equation 6 becomes:

$$
\Phi=\int_{0}^{\infty} Q(\lambda) a_{\mathrm{C}, \text { cornea }}(\lambda) \mathrm{d} \lambda .
$$

The collecting areas of mouse cones have not been estimated previously. We obtained the estimate $a_{\mathrm{C}}\left(\lambda_{\max }\right)=2.4 \mu \mathrm{m}^{2}$ based largely on information from the anatomical investigation of Carter-Dawson and LaVail (1979a), as follows. Mouse cone outer segments were assumed to have length $L=13 \mu \mathrm{m}$ and diameter $d=1.5 \mu \mathrm{m}$ at their base, tapering to 1.0 $\mu \mathrm{m}$ at their tips; in contrast rod outer segments were assumed to have length $L=25 \mu \mathrm{m}$ and uniform diameter $d=1.8 \mu \mathrm{m}$. Mouse cone inner segments were assumed to have a maximal diameter of $4 \mu \mathrm{m}, 1.8$-fold greater than mouse rod inner segments, $2.0 \mu \mathrm{m}$. The twofold greater maximal inner segment width of the cones produces a geometrical cross section fourfold larger for guiding light to the outer segment, on the assumption that all light entering the waveguide can be effectively funneled to the outer segment: thus, in Equation $5, f=(4 / 1.5)^{2}=7$ for cones and $f=(2 / 1.8)^{2}=1.24$ for rods. Rod and cones were assumed to have the same specific (optical) density at their respective $\lambda_{\max }$ values: $\Delta D\left(\lambda_{\max }\right)=0.015$ o.d. $/ \mu \mathrm{m}$. The quantum efficiency of photoisomerization was assumed to be the same for rods and cones, $\gamma=2 / 3$. Combining these factors in Equations 4 and 5, one obtains $a_{\mathrm{C}}\left(\lambda_{\max }\right)=1.3 \mu \mathrm{m}^{2}$ for rods and $a_{\mathrm{C}}\left(\lambda_{\max }\right)=2.4 \mu \mathrm{m}^{2}$ for cones. [For further discussion of the rod collecting area, see Lyubarsky and Pugh (1996). We defer analysis of the cone collecting area "at the cornea" to Discussion.]

Normalized spectral sensitivity. It is useful to define the normalized spectral sensitivity at the cornea of a photoreceptor class with peak sensitivity at $\lambda_{\max }$ in terms of collecting areas; thus, from Equations 4 and 5:

$$
\bar{S}_{\text {theory }}(\lambda) \equiv \frac{a_{\mathrm{C}, \text { cornea }}(\lambda)}{a_{\mathrm{C}, \text { cornea }}\left(\lambda_{\max }\right)}=\frac{\tau(\lambda)\left[1-10^{-\Delta D(\lambda) L}\right]}{\tau\left(\lambda_{\max }\right)\left[1-10^{-\Delta D\left(\lambda_{\max } L\right.}\right]} .
$$

Expressed in these terms, it is clear that provided the spectral maximum $\lambda_{\max }$, the normalized absorbance function $\Delta D(\lambda) / \Delta D\left(\lambda_{\max }\right)$, and the ocular media transmissivity $\tau(\lambda)$ are specified, only $D_{\max }=\Delta D\left(\lambda_{\max }\right) L$, the total axial density at the $\lambda_{\max }$, remains to be specified to determine the normalized spectral sensitivity.

ERG a-wave as a measure of the rod circulating current and rod action spectrum measurements. The primary goal of this investigation has been the characterization of murine cone-driven responses. However, because rod-driven ERG responses have been much more thoroughly quantified than those of cones, we have found it invaluable to use the rod a-wave as a benchmark for both spectral sensitivity analysis and quantification of the relative magnitudes of cone-driven signals. Analysis of the rod a-wave rests on the biophysical fact that the massed circulating currents of the rods give rise to a dipole layer field potential across the retina (Hagins et al., 1970), a potential that "depolarizes" the cornea in direct proportion to the average rod circulating current. The "a-wave" is the transient electrical potential resulting from suppression of some or all of the circulating current. Thus, if at any instant a brief flash sufficiently intense to saturate the a-wave amplitude is delivered, then the saturated value $a_{\max }$ satisfies:

$$
a_{\max } \propto J_{\text {circ }},
$$

where $J_{\text {circ }}$ is the rod circulating current at the moment immediately preceding the flash. Equation 9 can be used to recover the dependence of the rod circulating current on steady illumination and the time course of its recovery after a flash (Lyubarsky and Pugh, 1996; Pepperberg et al., 1996). It can also be combined with a well established model of the activation phase of the rod phototransduction cascade to predict the kinetics and sensitivity of the transient a-wave, $a(t)$, according to:

$$
\begin{aligned}
1-\left[\frac{a(t)}{a_{\mathrm{max}}}\right] & =F(t) \\
& =\exp \left[-1 / 2 \Phi A\left(t-t_{\mathrm{eff}}^{\prime}\right)^{2}\right], t>t_{\mathrm{eff}},
\end{aligned}
$$

(Lamb and Pugh, 1992; Breton et al., 1994; Lyubarsky and Pugh, 1996). In Equation $10, F(t)=J_{\text {circ }}(t) / J_{\text {circ,dark }}$, the circulating current normalized by its dark magnitude, $\Phi$ is the number of photoisomerizations produced by the flash, $A\left(\mathrm{~s}^{-2}\right)$ is the "amplification constant," and $t^{\prime}$ eff is a brief delay $(2-3 \mathrm{msec})$.

In this investigation we combined Equations 6 and 10 to determine for the first time the action spectrum between 340 and $600 \mathrm{~nm}$ of the dark-adapted murine a-wave. Thus, for a monochromatic flash of wavelength $\lambda$ producing a photon density $Q(\lambda)$ at the cornea, the predicted time course of the rod a-wave is:

$$
1-\left[\frac{a(t)}{a_{\max }}\right]=\exp \left[-1 / 2 Q(\lambda) a_{\mathrm{C}, \mathrm{cornea}}(\lambda) A\left(t-t_{\mathrm{eff}}^{\prime}\right)^{2}\right] .
$$

The protocol for determining the rod action spectrum used Equation 11, as follows. First, a family of ERGs was obtained in response to a series of $\lambda_{\text {nom }}=500 \mathrm{~nm}$ flashes, with intensities chosen to elicit a-waves whose maximum amplitude (i.e., amplitude at time of b-wave intrusion) was $15-50 \%$ of the saturated a-wave amplitude; a flash producing $\Phi=$ 280,000 was used to estimate $a_{\max }$. The normalized a-waves were later fitted as an ensemble with Equation 10 to estimate $A$ at $500 \mathrm{~nm}$ (Breton et al., 1994; Lyubarsky and Pugh, 1996). To determine rod sensitivity at any other wavelength $\lambda$, a series of flashes of graded intensity $Q(\lambda)$ was then delivered that produced a-waves of $15-50 \%$ saturated amplitude. With $A$ fixed (as required by the univariance of phototransduction) at its value at $500 \mathrm{~nm}$, the ensemble fitting of Equation 11 to the the a-wave data obtained with light of another wavelength $\lambda$ yielded an estimate of the product $a_{\mathrm{C} \text {,cornea }}(\lambda)=a_{\mathrm{C} \text {,cornea }}\left(\lambda_{\text {max }}\right) \bar{S}_{\text {theory }}(\lambda)$. Thus, with $a_{\mathrm{C} \text {,cornea }}\left(\lambda_{\text {max }}\right)$ fixed, we obtained an estimate of $S_{\text {theory }}(\lambda)$, the rod spectral sensitivity at this wavelength at the cornea (Eq. 8). We emphasize that the rod action spectrum provided a benchmark against which we gauged certain features of the cone action spectra, as well as a general control experiment for all spectral sensitivity determinations.

The reader may wonder why we measured the rod action spectrum with a-wave responses rather than with the more easily measured scotopic b-wave. One reason that we decided to estimate the rod spectrum from a-wave data is that the flash intensities involved were similar to those used to measure the cone-driven responses; this allowed use of the same filters and flash units. A second reason is that we feared that the scotopic b-wave might possibly be contaminated in the UV region of the spectrum by the relatively sensitive UV cone-driven b-wave.

Cone-driven signal isolation. Previous work has shown that cone-driven "b-waves" can be recorded reliably from mice in response to flashes (Peachey et al., 1993), and here we build on these previous findings. Nonetheless, proof of cone-signal isolation requires a valid monitor of the rod contribution to any response. This requirement is all the more acute in mice, because one of the cone-visual pigments is spectrally very similar to rhodopsin (Chiu et al., 1994). Because of the large ratio of rods to cones [ 30:1 (Carter-Dawson and LaVail, 1979a,b)], the saturated amplitude of the a-wave of the flash-ERG can be expected to monitor almost exclusively the rod circulating current at the moment of a probe flash (Eq. 9). However, because the efficacy of using the a-wave amplitude as monitor of the rods is limited by any cone contributions to the a-wave, we will present new evidence concerning the utility and limitations of this monitor in mice. In particular, we will estimate the magnitude and activation time course of a putative cone-driven a-wave and compare its features with those of the total (rod + cone) a-wave.

We used two protocols to isolate cone-driven ERG responses. In the first, the eye was exposed to $2.5 \mathrm{sec}$ steps of $520 \mathrm{~nm}$ background light of intensities estimated to produce $\varphi=3000-6000$ photoisomerizations/sec per rod; a probe flash of controlled intensity and spectral composition was delivered $2 \mathrm{sec}$ after the onset of the background. The second approach used an intense conditioning flash that suppressed the rod circulating current for several seconds; a probe flash then tested for cone-driven responses at various times after the conditioning flash before any rod signal recovery.

Measurement of cone action spectra from cone-driven b-waves. Conedriven b-waves, isolated as just described, were recorded in response to flashes of varying wavelengths and intensities. As we shall show in Results, for flashes of intensities that elicited responses $\leq 20 \%$ of the saturating magnitude, the responses were linear in flash intensity, i.e.:

$$
b(Q(\lambda), t)=Q(\lambda) S_{\mathrm{obs}}(\lambda) \bar{b}(t),
$$

where $b(Q(\lambda), t)$ is the appropriately defined cone b-wave response elicited by a flash of wavelength $\lambda$ and intensity $Q(\lambda), S_{\text {obs }}(\lambda)$ [nV (photon $\left.\mu \mathrm{m}^{-2}\right)^{-1}$ ] is the observed absolute sensitivity of the cone-driven ERG at wavelength $\lambda$, and $\bar{b}(t)$ is a scaled "dim-flash" response having unity 
amplitude at its peak. The obedience of cone b-wave responses to Equation 12 is a sufficient condition for the flash method to yield unique spectral sensitivity functions for the cones.

Two qualifications to the interpretation of the function $\bar{b}(t)$ in Equation 12 need be added. The first qualification concerns the presence of oscillatory potentials. In our analysis we will interpret $\bar{b}(t)$ as the function extracted from the cone-driven responses after removal of oscillatory potentials by appropriate filtering. The second qualification concerns shape differences in $\bar{b}(t)$ that may arise depending on the cone type in which the driving signals originate; thus, $\bar{b}(t)$ does not need to be identical in shape for signals driven by different classes of cones. An issue related to the second qualification concerns the use of a "white" flash to elicit saturating responses. This use was dictated by the limited energy available for waveband-limited flashes and the need to drive into saturation the responses of all $b$-wave generators receiving input from the UV- and/or M-cones. The problem of assessing the cone type of origin of cone-driven signals will be a principal focus of Results.

Because anesthesia limited the duration of the experiments, and because of the consequent need to use a number of different animals to get adequate numbers of rod- and cone-driven responses over the whole spectrum, we found it convenient to adopt $\lambda_{\text {nom }}=500 \mathrm{~nm}$ as a standard for spectral sensitivity measurements of both rod- and cone-driven responses. Thus, we always made measurements at $500 \mathrm{~nm}$ on each animal. Furthermore, throughout each experiment at intervals of 5-6 min a saturating white flash was delivered to monitor any secular changes in maximal signal amplitude; the maximal amplitude was found to vary no more than $10 \%$ between such flashes and no more than $30 \%$ over the time course of an entire experiment. Dim-flash responses were scaled relative to the amplitude of the nearest saturating response for the final analysis of sensitivity.

Template analysis of rod and cone action spectra. Characterization of the action spectra of photoreceptors has been greatly advanced by the application of photopigment template curves (Dartnall, 1953; Mansfield, 1985; Lamb, 1995). Such templates predict the shape of the normalized absorbance (low pigment density) spectrum. We fitted our action spectra with Equation 8, with the normalized absorbance spectrum given by Lamb's (1995) template 2; i.e., we assumed:

$$
\frac{\Delta D(\lambda)}{\Delta D\left(\lambda_{\max }\right)}=\frac{1}{\exp [a(A-x)]+\exp [b(B-x)]+\exp [c(C-x)]+D}
$$

where $\Delta D$ is specific density (compare Eq. 8), $\lambda$ is wavelength, $x=$ $\lambda_{\max } / \lambda$, and the parameter values are $a=70, b=28.5, c=-14.1, A=$ $0.88, B=0.924, C=1.104$, and $D=0.655$. The template analysis of action spectra data obtained "at the cornea" requires assumptions about the prereceptor media transmissivity $\tau(\lambda)$. We used values of $\tau(\lambda)$ obtained by Alpern et al. (1987) for rats, slightly modified as follows. The absorption for the neural retina in the mouse was assumed to be the same as that in rat, whereas absorption by the lens, which occupies most of the preretinal optical path in the mouse (Remtulla and Hallett, 1985), was taken as half of its value in rat. The prereceptor spectral transmissivity function $\tau(\lambda)$ obtained in this way was found to be well approximated by the expression:

$$
\tau(\lambda)=0.5+0.0018(\lambda-410) \text { for } \lambda>410 \mathrm{~nm} .
$$

where $\lambda$ is given in nanometers. Below $410 \mathrm{~nm}$ we did not apply a correction for prereceptor media, because the Alpern et al. (1987) transmission data extend only to $410 \mathrm{~nm}$.

Graphical presentation of action spectra data. For the analysis and presentation of action spectra data obtained with interference filters, each filter was assigned an effective wavelength, $\lambda_{\text {eff }}$, which could differ from the measured filter spectral density median, $\lambda_{\text {med }}$ (Fig. 1). The basis for this assignment is the principle of Univariance, which dictates that only the number of photoisomerizations generated by the flash in a given type of photoreceptors, and not their wavelength distribution, determines the response. Resting on this principle, we defined $\lambda_{\text {eff }}$ as the median wavelength of the integral specifying the total number of photoisomerizations produced by a source/filter combination. Thus, for an interference filter whose spectral transmission median in the apparatus is $\lambda_{\text {med }}$ and whose transmission spectrum is $T_{\lambda_{\text {med }}}(\lambda)$, we defined $\lambda_{\text {eff }}$ by the equation:

$$
\begin{aligned}
\frac{1}{2} \Phi_{0} & =a_{\text {C,cornea }}\left(\lambda_{\text {max }}\right) \int_{0}^{\lambda_{\text {eff }}} E_{\text {source }}(\lambda) T_{\lambda_{\text {med }}}(\lambda) \bar{S}_{\text {theory }}(\lambda) \mathrm{d} \lambda \\
& =a_{\text {C,cornea }}\left(\lambda_{\text {max }}\right) \int_{\lambda_{\text {eff }}}^{\infty} E_{\text {source }}(\lambda) T_{\lambda_{\text {med }}}(\lambda) \bar{S}_{\text {theory }}(\lambda) \mathrm{d} \lambda .
\end{aligned}
$$

Here $\Phi_{0}$ is the total number of photoisomerizations produced by the unattenuated flash in the receptors of the given type, $E_{\text {source }}(\lambda)$ is the quantal flux density of the unattenuated, unfiltered source at the cornea, $T_{\lambda_{\text {med }}}(\lambda)$ is the transmission spectrum of the particular filter having transmission median $\lambda_{\text {med }}$, and $a_{\mathrm{C}, \text { cornea }}\left(\lambda_{\text {max }}\right)$ is the collecting area of a particular class of photoreceptors at the cornea, and $\bar{S}_{\text {theory }}(\lambda)$ is the theoretical spectral sensitivity given by Equation 8 . Figure $1 B$ shows the application of Equation 15: it can be seen that whereas the lack of beam collimation alone shifted the median wavelength from 600 to $589 \mathrm{~nm}, \lambda_{\text {eff }}$ was also predicted to be shifted another $6 \mathrm{~nm}$, to $583 \mathrm{~nm}$, for rods with rhodopsin $\lambda_{\max }$ at $498 \mathrm{~nm}$. This $6 \mathrm{~nm}$ shift was the largest we found for the combination of filters and estimated pigment absorption spectrum, and in general shifts of such magnitude were only predicted to occur in the long-wave regions of asymptotic slope of the visual pigment absorption spectra. For all other regions of the photopigment absorption spectra, the pigment-specific shifts were calculated be $<2 \mathrm{~nm}$.

For the analysis and presentation of action spectra data one must assign the measured light $Q\left(\lambda_{\text {med }}\right)$ of Equation 1 to a single wavelength [ $\lambda_{\text {med }}$ has been substituted for $\lambda_{\text {nom }}$, because of the analysis of the filter transmission characteristics in situ (compare Fig. 1)]. Our assignment of the measured light to $\lambda_{\text {eff }}$ in Equation 15 is equivalent to making the approximation:

$$
Q\left(\lambda_{\text {med }}\right) \bar{S}\left(\lambda_{\text {eff }}\right) \approx \int_{0}^{\infty} E_{\text {source }}(\lambda) T_{\lambda_{\text {med }}}(\lambda) \bar{S}_{\text {theory }}(\lambda) \mathrm{d} \lambda
$$

where $Q\left(\lambda_{\text {med }}\right)$ is the measured quantity described in Equation $1, \lambda_{\text {eff }}$ is given by Equation 15 , and $\bar{S}_{\text {theory }}(\lambda)$ is the normalized spectral sensitivity given by Equation 8 . In summary, Equation 16 says that the measured quantal flux $Q\left(\lambda_{\text {med }}\right)$ at the position of the cornea is treated as if it were all concentrated at wavelength $\lambda_{\text {eff }}$, where $\lambda_{\text {eff }}$ has the property that the theoretically computed contributions to the photoreceptor's total quantum catch from light below and above $\lambda_{\text {eff }}$ in the spectral distribution at the retina are equal (Eq. 15).

Rod a-wave as a gauge for estimating magnitudes of generator currents of other retinal cell types. On the basis of the now certain identification of the rod circulating current as the generator of the field potential whose suppression is the dark-adapted a-wave, the saturated amplitude of the dark-adapted a-wave can provide a gauge for estimating from field potentials the underlying generator currents of other retinal cell types. To use this gauge, the proportionality relation of Equation 9 must be expressed more completely. The corneally recorded potential and the generator current of a specific radially oriented retinal cell type can be shown to obey approximately the following relationship (Hagins et al., 1970; Pugh et al., 1998):

$$
\Delta V_{\text {cell,cornea }}=V_{\text {cornea }}-V_{\text {ref }}=c_{\text {electrodes }} \rho_{\text {cell }} J_{\text {cell }} \bar{r}_{\text {layer }} .
$$

In Equation $17 \Delta V_{\text {cell,cornea }}(V)$ is the electrical potential between corneal and reference electrodes caused by complete activation (or inactivation) of the generator current of a particular retinal cell type, $c_{\text {electrodes }}$ is a dimensionless factor attributable to electrode type and placement, $\rho_{\text {cell }}$ (cells $\mathrm{cm}^{-2}$ ) is the spatial density of the cell type in the retina, $\bar{r}_{\text {layer }}\left(\Omega \mathrm{cm}^{2}\right)$ is the effective or "average" resistance of the retinal layer in which the cell is found, and $J_{\text {cell }}(A)$ is the generator current; the product $\rho_{\text {cell }} J_{\text {cell }}$ is the maximal radial current density generated by the simultaneous activity of all the cells of the specific type. In general, we can eliminate common terms in Equation 17 that arise from retinal and recording geometry by forming a ratio for two cell types. Specifically, 

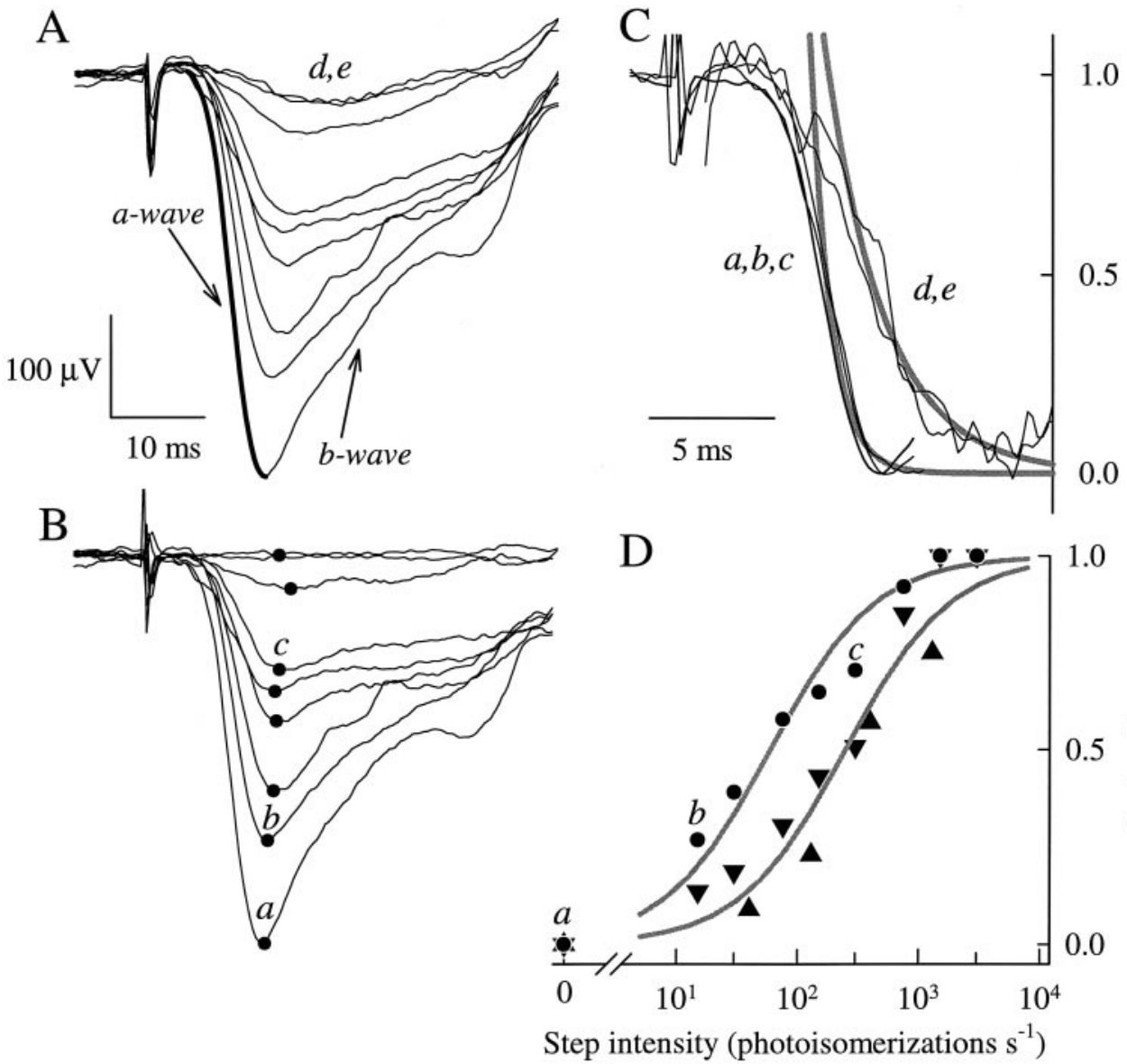

Figure 2. Separation of cone from rod signals by steady backgrounds. $A$, ERGs elicited by a white flash calculated to produce $\Phi=280,000$ photoisomerizations/rod, delivered in the dark, and in the presence of a series of $500 \mathrm{~nm}$ steps of light (backgrounds) generating $\varphi=$ $15,30,76,150,300,760,1500$, and 3020 photoisomerizations/sec per rod, respectively; the amplitude of the responses diminishes as the background intensity increases. The traces are averages of two to three responses, and the flashes were presented $2 \mathrm{sec}$ after the onset of the light steps. The a-wave is the initial, corneal-negative response component: the a-wave trace obtained for the dark-adapted condition is shown thickened. The b-wave is the cornealpositive-going ERG component that follows and effectively truncates the a-wave. $B$, ERGs from $A$ after subtraction of the average of the traces $(d, e)$. The filled circle attached to each trace is its minimum (except for the topmost traces, where the circle is plotted at the average time of the other circles). The time and amplitude scales of $B$ are the same as for $A$. $C$, Traces $a, b, c$ from $B$ and $d, e$ from $A$ normalized. The terminal portions of the traces have been fitted with decaying first-order exponential functions (thickened gray curves): the exponential through traces $a, b, c$ has a time constant of $0.83 \mathrm{msec}$, whereas that through traces $d, e$ has a time constant of $2.5 \mathrm{msec}$ (trace $c$ was shifted leftward by $0.6 \mathrm{msec}$ to emphasize the common shape of the terminal portions of $a-c) . D$, Inferred steady "photocurrent" response amplitudes of rods to the background light steps, derived from the data of $B(\mathbf{0})$ and from data of two other mice $(\boldsymbol{\Lambda}, \boldsymbol{\nabla})$. Each set of symbols gives the maximum amplitudes of the "cone-corrected" a-wave responses, as shown in $B$, by application of Equation 20 . Note the exact amplitude correspondence between the circles plotted in $B$ and $D$ : the labels $a$, $b$, and $c$ from $B$ have been carried over to $C$ to show the correspondence. The smooth curves are hyperbolic or "Naka-Rushton" saturation functions (Eq. 21), with semisaturation constants of 60 and $250 \varphi$. because of the identification of the rod circulating current as the generator of the dark-adapted a-wave we have the following:

$$
\frac{\Delta V_{\text {cell }}}{\Delta V_{\text {rods }}}=\frac{\Delta V_{\text {cell }}}{a_{\text {max,rods }}}=\frac{\rho_{\text {cell }}}{\rho_{\text {rods }}} \frac{J_{\text {cell }}}{J_{\text {circ,rods }}} \frac{\bar{r}_{\text {cell layer }}}{\bar{r}_{\text {rod layer }}}
$$

where we have dropped the subscript "cornea" for simplicity. In our application of Equation 18, the potentials of the left-hand side will be taken from the ERG data, and we will assume that the layer resistances, which are the products of the interstitial resistivities and layer thickness, are roughly equal for the outer nuclear and inner nuclear layers. To apply Equation 18, two quantities characterizing rods are needed: (1) $\rho_{\text {rods }}$, the spatial density of rods in the retina, and (2) $J_{\text {circ,rod, }}$ the average rod circulating current.

We take the average rod circulating (dark) current to be $25 \mathrm{pA}-\mathrm{a}$ reasonable compromise between the early estimate of $70 \mathrm{pA}$ by Hagins et al. (1970) and more recent suction pipette measurements of mammalian rods (Baylor et al., 1984; Nakatani et al., 1991; Kraft et al., 1993). Estimates of rod density in rodent retinas converge on the value of $3 \times$ $10^{7}$ rods $\mathrm{cm}^{-2}$ (cf. Reiser et al., 1996). Thus, the maximal radial current density attributable to rod circulating current is $\rho_{\text {rods }} J_{\text {circ,rods }}=25 \times$ $10^{-12} \times 3 \times 10^{7}=750 \times 10^{-6} \mathrm{~A} \mathrm{~cm}^{-2}$; this maximal current density occurs at the retinal layer corresponding to the junction of the rod outer and inner segments, because the circulating current sources are all in the rod inner segment membrane and the sinks are in the outer segment membrane (Hagins et al., 1970; Pugh et al., 1998). Assuming that the layer resistances through which flow the signal currents of other radially oriented retinal cell types are comparable, the "rod gauge" (Eq. 18) indicates that a radial current density in the mouse retina of $750 \mu \mathrm{A}$ $\mathrm{cm}^{-2}$ gives rise to a potential between the cornea and reference elec- trode of $\sim 400 \mu \mathrm{V}$, the value of $a_{\text {max, rod }}$ [see Table 1; see also Lyubarsky and Pugh (1996)].

\section{RESULTS \\ Isolation of cone-driven components of the murine ERG: cone a-wave}

Figure $2 A$ shows a family of ERGs elicited with a white flash estimated to produce $\Phi \approx 2.8 \times 10^{5}$ photoisomerizations/rod, sufficiently intense to saturate the a-wave amplitude (Lyubarsky and Pugh, 1996). The flash was delivered in the fully darkadapted condition and in the presence of $520 \mathrm{~nm}$ background steps whose intensity was varied to produce from $\varphi=15-3020$ photoisomerizations/sec per rod. Each increase in the intensity of the step up to approximately $\varphi=1500$ further decreased the amplitude of the a-wave response to the flash; however, as shown by the coincidence of traces $d$,e , the increase from $\varphi=1500$ to 3020 had no effect on the amplitude. Rather, the flash now evoked a residual a-wave whose amplitude $(25 \mu \mathrm{V})$ remained at $6-7 \%$ of the amplitude $(395 \mu \mathrm{V})$ of the dark-adapted response. In 12 mice exposed to background steps of $\varphi=3000-6000$, the residual a-wave amplitude evoked by intense white flashes such as in Figure $2 A$ was $21 \pm 10 \mu \mathrm{V}$ (mean $\pm \mathrm{SD})$.

The residual a-wave component measured in the presence of such backgrounds was also significantly slower in its "rise" than the a-wave elicited from the dark-adapted retina: the rising phase 


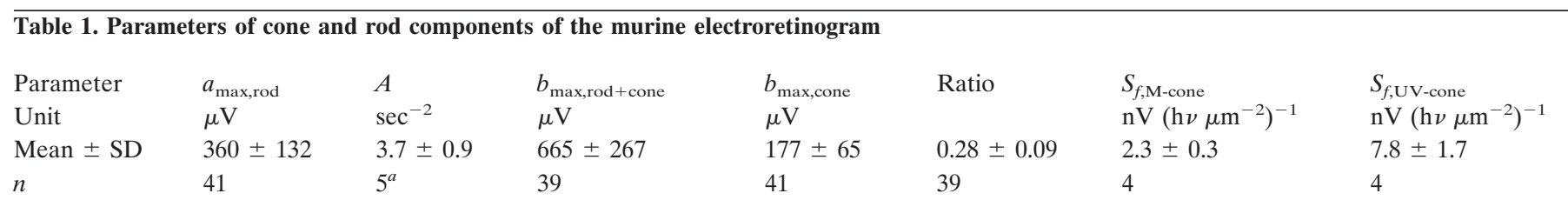

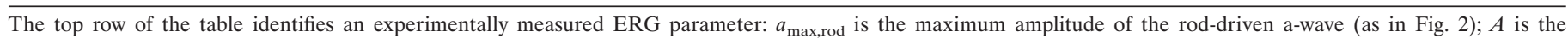

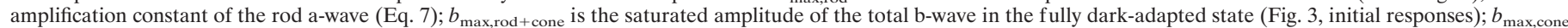

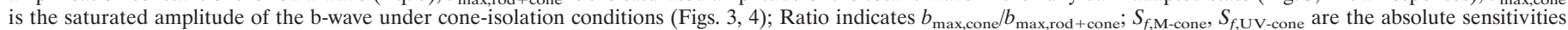
at the cornea of the cone-driven b-wave for 500 and $340 \mathrm{~nm}$ flashes (Figs. 4, 5). $n$ is the number of animals for which the measurement was made.

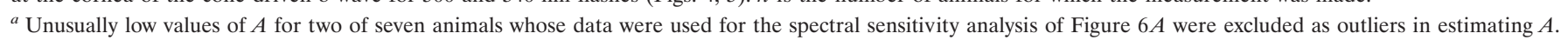

of the saturated dark-adapted a-wave has a time to peak of $\sim 8$ msec, whereas the residual a-wave reaches its peak amplitude in $\sim 14$ msec. Further evidence for this relative slowness is presented in Figure $2 C$, in which the normalized traces $a-c$ of $B$ and of the normalized residual traces $d$, $e$ of $A$ have been replotted and fitted with exponentials having time constants of 0.83 and 2.5 msec, respectively. As an alternative analysis to the fitting of decaying exponential functions, we applied to the a-wave traces obtained in response to the saturating flash the model of the a-wave presented by Breton et al. (1994), as modified by Smith and Lamb (1997) to incorporate analytically the effect of the photoreceptor membrane time constant. For nine sets of responses from different mice analyzed in this manner, the estimated time constant was $1.0 \pm 0.1 \mathrm{msec}($ mean $\pm \mathrm{SD})$ for the terminal phase of the dark-adapted a-wave response, and $2.9 \pm$ $0.8 \mathrm{msec}$ for the residual a-wave recorded in the presence of a background estimated to produce either 3020 or 6000 photoisomerizations/sec per rod. These different time constants are consistent with rod and cone origins, respectively. Specifically, the "rise time" of circulating current responses to intense flashes are rate-limited by the cell membrane time constant $\tau_{\text {cell }}$ (Penn and Hagins, 1972; Cobbs and Pugh, 1987), which have been estimated for mammalian photoreceptors to be $\tau_{\text {rod }} \approx 1 \mathrm{msec}$ (Penn and Hagins, 1972; Schneeweis and Schnapf, 1995), and $\tau_{\text {cone }} \approx 2-4$ msec (Schneeweis and Schnapf, 1995). Analyses of saturating human rod and cone a-waves have yielded similar estimates: $\tau_{\text {rod }}$ $\approx 1 \mathrm{msec}$ and $\tau_{\text {cone }}=2-4 \mathrm{msec}$ (Hood and Birch, 1993, 1995; Cideciyan and Jacobson, 1996; Smith and Lamb, 1997).

In sum, based on its electrical sign, its relative insensitivity to background light, and the relative sluggishness in its rise, we attribute the residual a-wave component (Fig. 2A, $C$, traces $d, e$ ) mainly to the suppression of the retinal field potential generated by the circulating currents of cone photoreceptors. In Figure $2 B$ we show the traces of Figure $2 A$ with the presumed cone a-wave component subtracted out, and in Figure $2 D$ we plot steady-state response-intensity relations for the rod photocurrent responses to the background steps, derived from the traces in Figure $2 B$ as follows.

The rod photocurrent response $R$ is the complement of the circulating current; i.e., the rod photocurrent evoked by a light step of intensity $\varphi$ necessarily satisfies:

$$
R(\varphi)=J_{\text {circ,dark }}-J_{\text {circ }}(\varphi) .
$$

Combining the defining relation (Eq. 19) with Equation 9, we arrive at the following expression for the normalized response to the background steps:

$$
\frac{R(\varphi)}{R_{\max }}=\frac{J_{\text {circ,dark }}-J_{\text {circ }}(\varphi)}{J_{\text {circ,dark }}}=\frac{a_{\text {max,dark }}-a_{\text {max }}(\varphi)}{a_{\text {max dark }}} .
$$

Thus, in Figure $2 D$ we plot $\left(a_{\text {max,dark }}-a_{\text {max }}(\varphi)\right) / a_{\text {max,dark }}$ as a function of the step intensity for the data of Figure $2 B$, along with results obtained in the same way from two additional mice. The conclusion that the data points represent the normalized amplitudes of rod photocurrent responses to the light steps is supported by their dependence on step intensity, which can be characterized with a hyperbolic saturation function:

$$
\frac{a_{\mathrm{max}, \mathrm{dark}}-a_{\max }(\varphi)}{a_{\mathrm{max}, \mathrm{dark}}}=\frac{\varphi}{\varphi_{1 / 2}+\varphi} .
$$

In Equation $21 \varphi_{1 / 2}$ is the half-saturating intensity, a measure of rod light sensitivity to the step intensity. For the animal whose data are shown in Figure $2 B(\mathbf{0}), \varphi_{1 / 2}=60$, whereas the responses of the other two animals are reasonably well characterized by Equation 21 with $\varphi_{1 / 2}=250$. This latter value is consistent with steady-state response versus intensity functions of isolated rods of a number of mammalian species (Nakatani et al., 1991), which have been characterized by hyperbolic saturation functions with $\varphi_{1 / 2}=150-300 \Phi /$ sec. Taking the value $\varphi_{1 / 2} \approx 250$ as a reasonably conservative figure [because the data tend to saturate somewhat more steeply than the hyperbolic relation (Eq. 21)], we conclude that $>92 \%$ of the rod circulating current should be suppressed for $\varphi=3020$. We thus chose to use backgrounds estimated to produce $\varphi=3000-6000$ as standard for cone signal isolation, with the goal of keeping the cone signaling pathways (especially those of the M-cones) maximally sensitive while simultaneously maximally suppressing rod-driven retinal activity. Below (see Figs. 4-6) we will present evidence that the b-waves recorded in the presence of such backgrounds are cone-driven. First, we show a second method of isolating cone-driven ERG responses, which provides converging evidence for such isolation.

\section{Isolation of cone-driven components of the murine ERG: cone b-wave}

In Figure 3 the top trace in the left-hand panel shows the ERG of a fully dark-adapted mouse obtained in response to an intense white flash estimated to produce $\Phi=1.5 \times 10^{6}$ photoisomerizations/rod. The subsequent nine traces show repeated responses to the same flash, and at various intervals the response to a second, white "probe" flash one-tenth as intense, delivered at the times indicated on the graph. The probe flash generates a cornealpositive (b-wave) response component that recovers rapidly, during a period when there is no reliably measurable change in the initial, corneal-negative a-wave.

The recovery time course of the peak amplitude of the b-wave response to the probe flashes is shown in the inset at the bottom right of Figure 3. Here it is seen that there is a rapid phase of b-wave recovery in the initial second after the conditioning flash, followed by a more slowly increasing corneal positivity. On the 


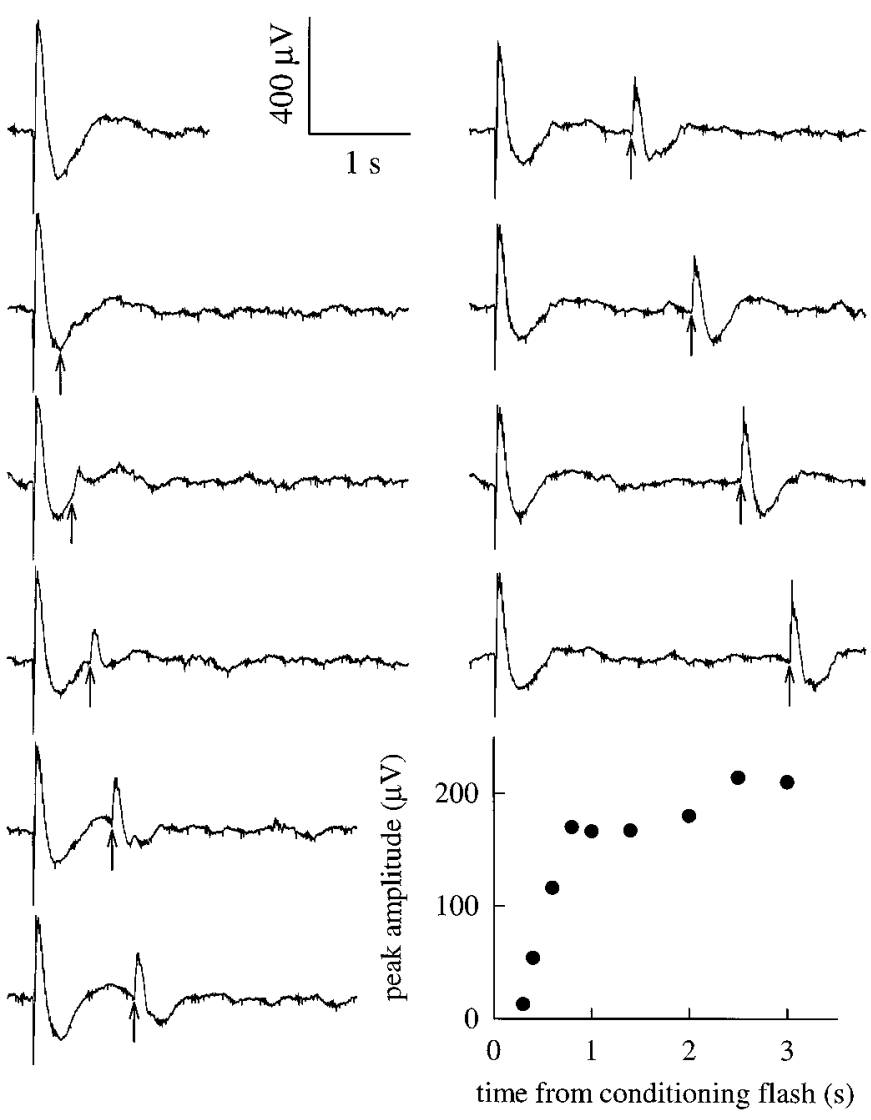

Figure 3. Separation of cone from rod signals by flashed adapting light. Isolation of cone-driven responses by a "double-flash" protocol. Ten traces are shown. Initially in each case a white conditioning flash producing $\Phi=1.5 \times 10^{6}$ photoisomerizations/rod was delivered to the darkadapted eye, producing an ERG having a 260-300 $\mu \mathrm{V}$ corneal-negative a-wave, followed immediately by a $530-660 \mu \mathrm{V}$ positive-going b-wave, and then a slower, negative-going potential. At the times indicated by the upward-pointing arrows, a second flash producing $\Phi=1.5 \times 10^{5}$ was delivered to probe recovery from the initial flash. Inset at bottom right, Symbols plot the peak amplitude of the cone-driven b-wave responses shown by the traces.

basis of its rapid recovery time course in the absence of an a-wave component exceeding a few percent of the initial (dark-adapted) $\approx 300 \mu \mathrm{V}$ a-wave, we attribute the corneal-positive response recorded in the $3 \mathrm{sec}$ period after the conditioning flash exclusively to cone-driven cells. In contrast, for the same intensity conditioning flash, the rod a-wave or circulating current remains in complete saturation for $>3 \mathrm{sec}$ and has a recovery half-time of $\sim 10$ sec [Penn and Hagins (1972), their Fig. 8]. In summary, this "double-flash" procedure provides a complementary method to the use of steady backgrounds for cone-signal isolation illustrated in Figure 2.

Table 1 presents summary statistics for some key quantities measured in experiments like those illustrated in Figures 2-3. One particularly interesting quantity is the ratio of the saturating b-wave amplitude obtained under cone-signal isolation conditions to that obtained in the dark-adapted state. In Figure 3, the peak amplitude of the cone-driven b-wave ( $b_{\text {max,cone }}$ ) is seen to be $220-250 \mu \mathrm{V}, \sim 35 \%$ of the amplitude of the dark-adapted b-wave $\left(b_{\text {max,rod }+ \text { cone }}\right), 660 \mu \mathrm{V}$. In similar experiments on 39 mice, we found the ratio $b_{\text {max,cone }} / b_{\text {max,rod }+ \text { cone }}$ to be $0.28 \pm 0.09$ (mean \pm $\mathrm{SD}$; Table 1), a result suggesting that cone-driven on-bipolar cells generate a maximal current density nearly half that of rod bipolars (Discussion).

The use of "white" flashes to generate cone-driven responses in experiments such as that of Figure 3 raises questions in light of the evidence that the mouse retina has both UV-and M-sensitive cones (see introductory remarks). For example, the question arises as to whether the UV- and M-cones are stimulated and adapted to the same degree by such white flashes, and in particular, whether the b-wave generators receiving input from each cone type are driven into saturation. We thus undertook experiments to measure the action spectrum and quantify the properties of cone-driven b-wave responses.

\section{B-wave response families under cone-isolation conditions}

Figure 4 illustrates two families of ERG responses of a single mouse to a series of flashes of nominal wavelengths $\lambda_{\text {nom }}=340$ and $520 \mathrm{~nm}$ of varying intensity under conditions of cone-signal isolation. These responses present a number of well known features of cone-driven ERG responses, including the progressive appearance of large oscillatory potentials (Heynen et al., 1985; Peachey et al., 1987).

We derived the action spectrum of cone-driven signals from data such as those illustrated in Figure 4. As discussed in Materials and Methods, a sufficient condition for the b-wave response to have a unique action spectrum is that it be linear in intensity over some range. Inspection of the traces in the top panel of Figure 4 leaves some doubt about linearity, inasmuch as the oscillatory potentials exhibit a strong nonlinearity, such that the time-to-peak of the individual oscillations depends on flash intensity even at relatively low intensities. We dealt with this difficulty by filtering the raw traces digitally with a Gaussian filter, thereby obtaining the smooth, thickened gray traces. Although the Gaussian has the defect of being an anticipating filter, it nonetheless provides a robust, nonsubjective method of extracting a "running mean" curve that does not exhibit the oscillations. The Gaussian-filtered traces are nearly invariant in shape at the lowest intensities, providing support for the derivation of a unique action spectrum (Eq. 12). A second benefit of applying this filter is that it gives by subtraction a useful representation of the oscillatory potentials, as seen in the bottom panels of Figure 4.

Figure $5 A$ shows response peak amplitude versus intensity functions derived from the filtered traces of Figure 4. In this double-log plot, response amplitude linearity is represented by lines of unity slope, which are shown drawn through the symbols representing the lowest amplitude responses. In Figure $5 B$ the data from $A$ have been replotted with respect to a normalized amplitude coordinate, along with data of five other animals obtained with the same and two other wavelengths. Since the work of Fulton and Rushton (1978), it has been traditional in presenting such data to fit them over the entire intensity domain with a single saturation function, such as the hyperbolic function shown in Figure $2 D$. We have not fitted these data with a specific saturation function for three reasons. First, in the UV region of the spectrum the energy of our flash lamps was insufficient to generate saturating responses. Second, the filtering is less defensible in the upper range of intensities where the oscillations are largest and where the detailed form of the saturation function's compressive nonlinearity affects the fitting. Third, and most importantly, as we shall show below, although driven by photons caught by an M- and a UV-cone pigment, the b-wave responses cannot be explained on the hypothesis that there are completely 
Figure 4. Response families of cone-driven b-waves for 340 and $500 \mathrm{~nm}$ flashes. Top panel, Black traces show the responses of a single mouse (no. 315) to a series of UV $\left(\lambda_{\text {nom }}=340 \mathrm{~nm}\right)$ and midwave $\left(\lambda_{\text {nom }}=520 \mathrm{~nm}\right)$ flashes; each trace is the average of from 5 to 40 individual responses. The smooth, thickened gray traces were obtained by digitally filtering the averaged responses with a Gaussian filter with $\sigma=8 \mathrm{msec}$ (bandwidth $16.6 \mathrm{~Hz}$ at 3 $\mathrm{dB}$ cutoff). The UV intensity series delivered (from lowest to highest intensity) $Q\left(\lambda_{\text {nom }}\right)=1110,2480,4390$, and 8800 photons $\mu \mathrm{m}^{-2}$ at the cornea, and the midwave series delivered 4550, 9090, 18700, 37500, and 75000 photons $\mu \mathrm{m}^{-2}$ at the cornea. The 4 msec segment of the traces immediately after the flash contained a flash artifact, and so this segment has been omitted for clarity; this segment was also set to zero before the Gaussian filter was applied. Bottom panel, Cone-driven oscillatory potentials extracted from the responses in the top panel by subtracting the Gaussian-filtered responses from the averaged responses; these difference traces are shown on the same time and amplitude scales as the responses in the top panel. The difference traces were digitally filtered with a Gaussian filter having a bandwidth of $133 \mathrm{~Hz}$.

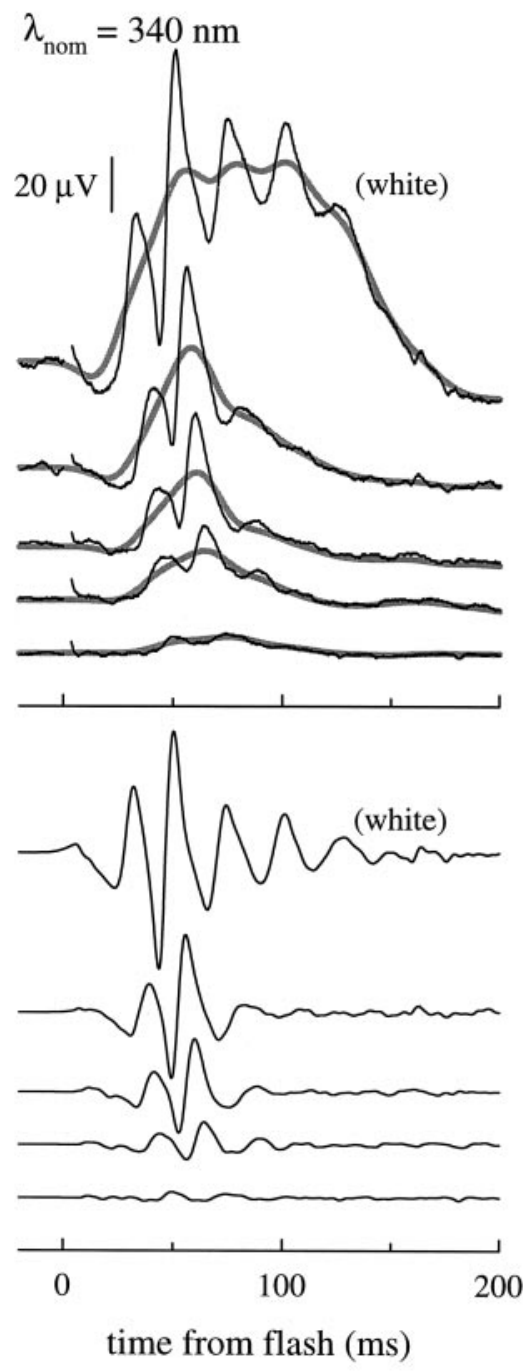

$\lambda_{\text {nom }}=520 \mathrm{~nm}$
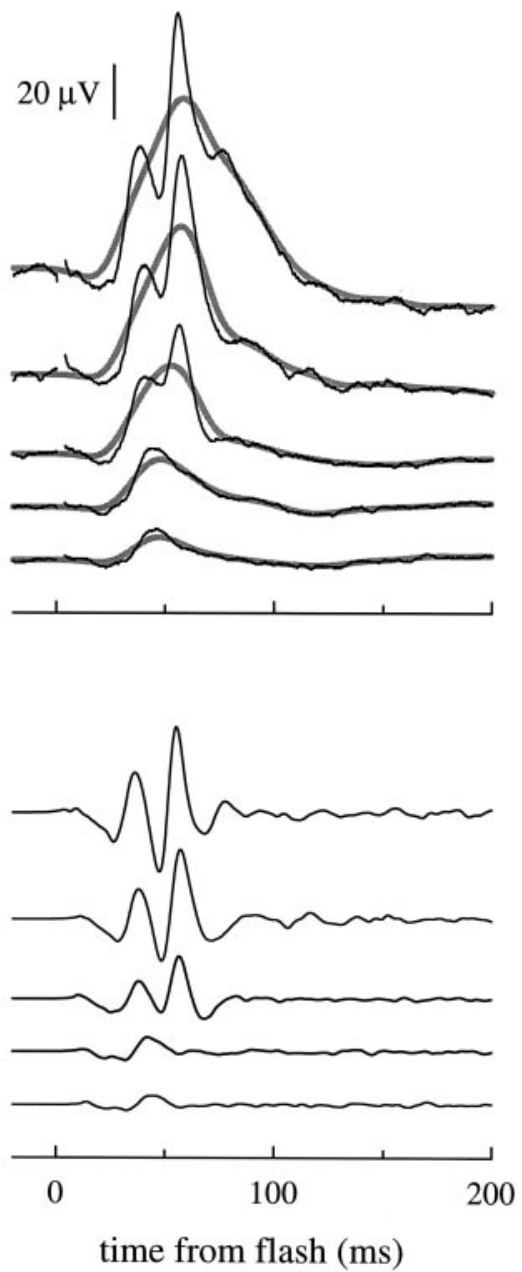

separate neural pathways for signals originating in each cone pigment. Thus, we would not expect any simple saturation function to describe the amplitude versus intensity function independent of wavelength.

It bears emphasizing that the absence of a simple functional description of cone b-wave saturation in the mouse in no way compromises the derivation of the action spectrum of the responses. Rather, as explained in Materials and Methods, a sufficient condition for determination of a unique spectral sensitivity is an intensity domain of response linearity. In Figure 5, such linearity is manifested in the intensity domains for each wavelength over which unity slope lines describe the data. In these domains of linearity the derived sensitivity measure is the ratio of the ordinate amplitude (in microvolts) divided by the abscissa (in photons $\mu \mathrm{m}^{-2}$ ). Before presenting the cone action spectrum derived from amplitude versus intensity data such as in Figure 5, we will first describe the rod action spectrum, which provides benchmark information about several features of the mouse eye media and retina.

\section{Rod a-wave action spectrum}

Figure $6 A$ presents the spectral sensitivity of the a-wave of fully dark-adapted mice over the spectral range 350-600 nm, derived with the analysis described in Materials and Methods. The mean amplification constant was $A_{\text {rod }}=3.7 \pm 0.9 \mathrm{sec}^{-2}$ (Table 1), $50 \%$ lower than that $\left(7.2 \pm 1.5 \mathrm{sec}^{-2}\right)$ estimated previously for C57BL/6 mice with a different method of delivering light (Lyubarsky and Pugh, 1996). We offer three possible explanations for the discrepancy in $A_{\text {rod }}$ between the studies: (1) the previous method of light delivery (through a fiber optic coupled to a contact lens) may have been more effective; in the present, more lengthy experiments, some corneal drying and opacification occurs; (2) an unaccounted-for systematic error in estimating light delivered to the retina from different angles in the old and new stimulation geometries; and (3) differences in the animals.

As expected for rhodopsin, the action spectrum derived from the dark-adapted a-wave data has a primary peak near $500 \mathrm{~nm}$ and a broad secondary maximum in the UV. We fitted the spectrum with the pigment template function (Eq. 13) adjusted for self-screening and corrected for the media transmissivity as described by Equation 8. The spectrum is reasonably well described by a retinal ${ }_{1}$ pigment with $\lambda_{\max }=498 \mathrm{~nm}$ and an end-on optical density of 0.3 (dark gray, smooth curve). The template pigment curve does not extend much below the pigment $\lambda_{\max }$, and in particular does not include the UV region of the spectrum. To derive a representation of the spectrum below $460 \mathrm{~nm}$, we fitted the data with a fifth-order polynomial (light gray curve), which was forced to agree with the template curve up to $500 \mathrm{~nm}$. 

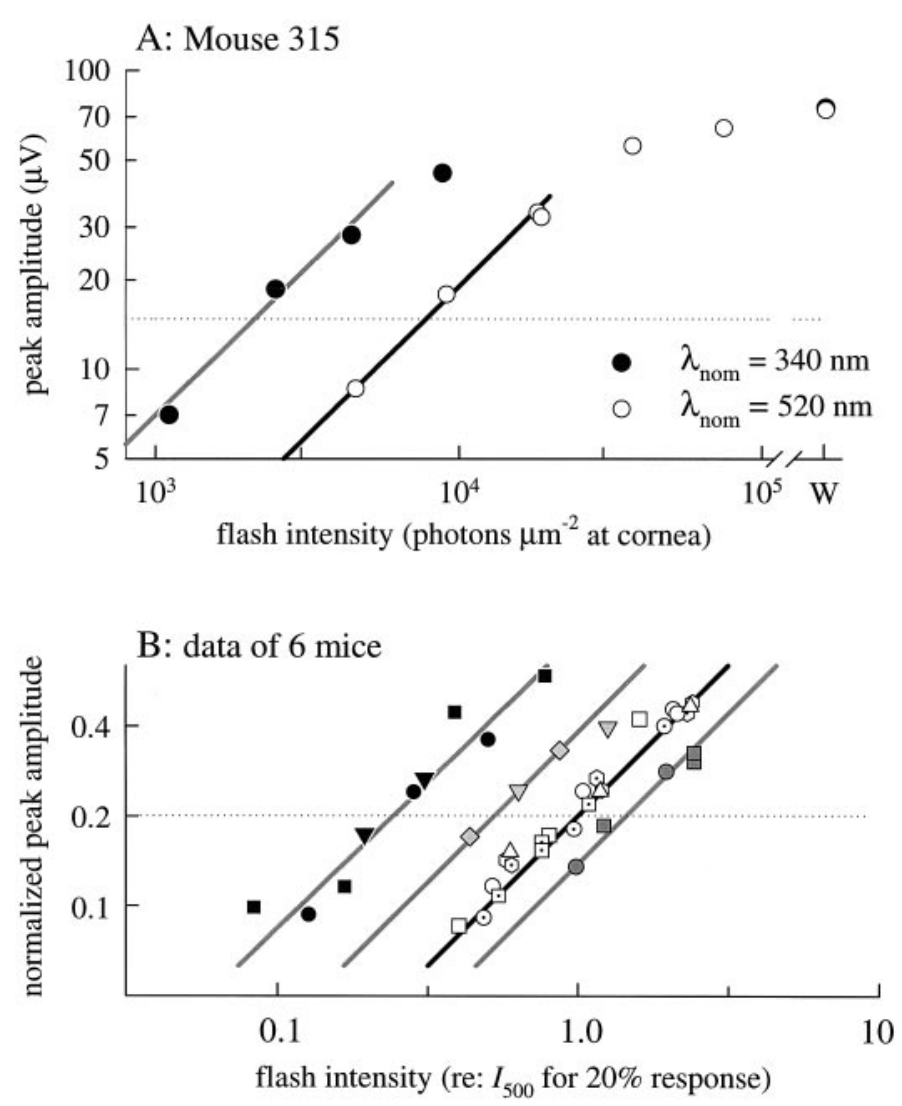

Figure 5. Response amplitude versus intensity functions for cone-driven b-waves. $A$, The peak amplitudes of the Gaussian-filtered traces of Figure 4 (mouse no. 315) are plotted as a function of the flash intensity in double-logarithmic coordinates. Lines of slope unity, representing linearity of the peak amplitudes, have been fitted to the three lowest points in each set. The dotted line corresponds to a peak response amplitude of $20 \%$ of the saturating amplitude. $B$, Amplitude versus intensity data for six mice obtained with flashes of five different wavelengths. Peak amplitudes such as those in $A$ were first normalized by dividing them by the saturating amplitude; then, the intensities were scaled by the intensity $I_{500}$, estimated (by interpolation) to produce $20 \%$ of the normalized amplitude for $\lambda_{\text {nom }}$ $=500 \mathrm{~nm}$ flashes for the individual mouse. Wavelength $\left(\lambda_{\text {nom }}\right)$ keys for the data are as follows (from left to right): $340 \mathrm{~nm}$ (black symbols), $400 \mathrm{~nm}$ (light gray symbols), $500 \mathrm{~nm}$ (symbols with dots in center), $520 \mathrm{~nm}$ (open symbols), $560 \mathrm{~nm}$ (dark gray symbols). Different-shaped symbols are used for the data of different mice. Note that the open and filled circles representing the data of mouse no. 315 from $A$ are replotted in $B$ and have the same relative locations on both $x$ - and $y$-axes (this serves to illustrate the method of $x$ - and $y$-scaling). (Data obtained at other wavelengths have been omitted for the sake of clarity. In the derivation of the cone b-wave action spectrum the response vs intensity data of each animal were regressed individually, as in $A$.)

\section{Cone b-wave action spectrum}

Figure $6 B$ presents the action spectrum for the cone-driven b-wave derived from the analysis illustrated in Figure 5. The spectrum, which represents data of seven mice, features two maxima: one at $\approx 510 \mathrm{~nm}$ in the visible spectrum and a second at $\approx 355 \mathrm{~nm}$ in the UV; these maxima clearly reflect the well established UV- and M-cone photopigments (Jacobs et al., 1991). The smoothed, dark gray curves were generated with Lamb's (1995) template (Eqs. 8, 13) with $\lambda_{\max }$ values at 355 and $508 \mathrm{~nm}$, respectively, and were fitted to the data by eye. Flash sensitivity near the UV maximum is $\sim 3.5$ - to fivefold higher than at the midwave maximum, based on three distinct but related criteria: (1) the relative ordinate positions of the template curves in Figure

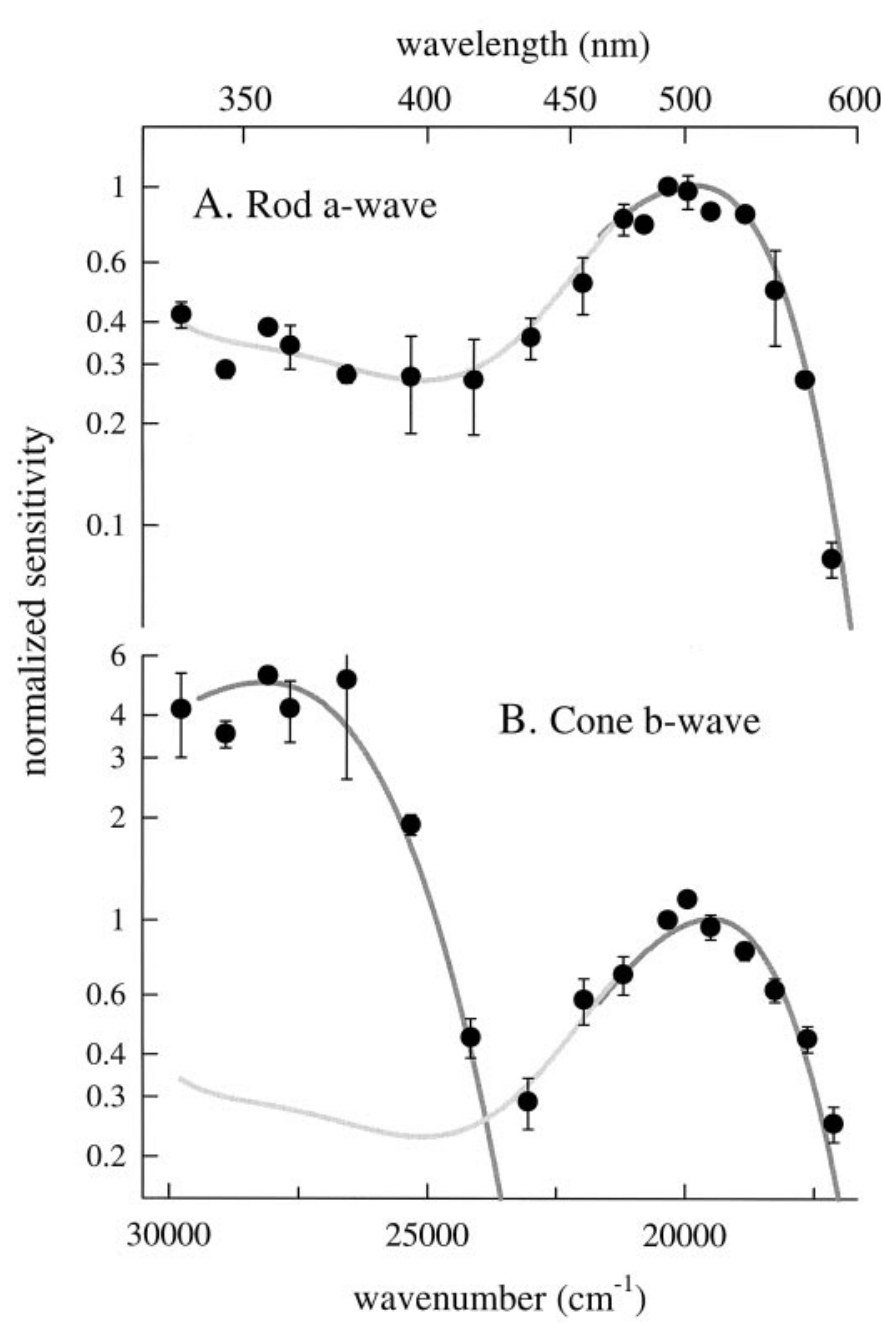

Figure 6. Spectral sensitivities of murine rods and cones derived from ERGs. $A$, Spectral sensitivity of the dark-adapted murine a-wave. Each point is the mean \pm SD of two to five determinations of the sensitivity of the a-wave at the cornea, estimated by application of Equation 11 to families of a-wave responses, as described in Materials and Methods. The data of each animal were individually normalized to a sensitivity of 1.0 at $500 \mathrm{~nm}$. The dark gray smooth curve (plotted from 460 to $600 \mathrm{~nm}$ ) was generated with Equations 8 and 13, with $\lambda_{\max }=498 \mathrm{~nm}$, and a maximum axial absorbance $D_{\max }=0.3$. The light gray curve drawn through the data below $460 \mathrm{~nm}$ is a fifth-order polynomial obtained by least-squares regression. $B$, Spectral sensitivity of the cone-driven b-wave. The data points are sensitivity estimates (mean $\pm \mathrm{SD}$ ) from two to five animals, each estimate having been derived from a response versus intensity function such as shown in Figure 7. The smoothed gray traces were generated with Equations 8 and 13, with $\lambda_{\max }=355$ and $508 \mathrm{~nm}$, respectively, and $D_{\max }=0.1$ (negligible self-screening). The light gray curve was obtained from the fifth-order polynomial describing the rod spectrum below $460 \mathrm{~nm}$ but has been scaled linearly by 0.85 as a correction for the absence of self-screening.

$6 B$, (2) the average of all data points near the two maxima, and (3) the ratio of sensitivity at $\lambda_{\text {nom }}=340 \mathrm{~nm}$ to that at $\lambda_{\text {nom }}=500$ $\mathrm{nm}$ for four mice that were each tested at both 340 and $500 \mathrm{~nm}$. The absolute sensitivities of the latter four mice at these two wavelengths are reported in Table 1 . Because the midwave maximum is located close to the rod action spectrum peak, it is reasonable to assume that the M-cone pigment is very similar to rhodopsin in its spectral properties. Therefore, the height of the $\beta$-band of the rod spectrum (Fig. $6 A$ ) provides an estimate for any contribution of the M-cone pigment to the peak UV sensitivity of 

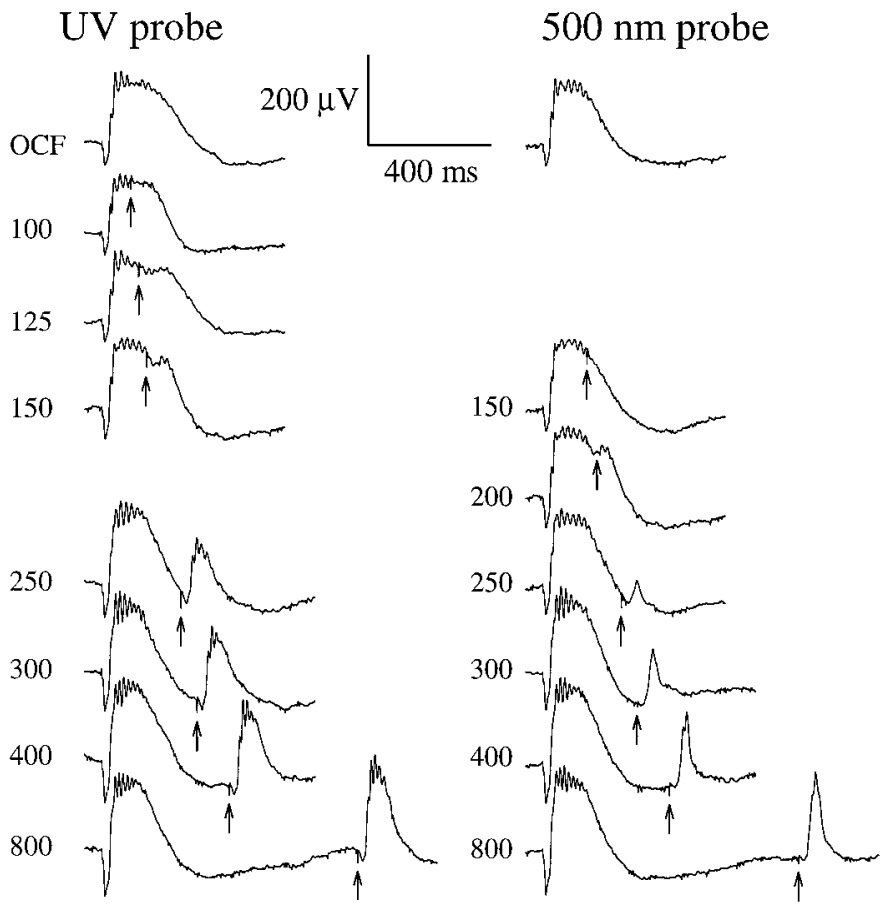

Figure 7. Interaction of signals originating in the UV- and M-cone pigments. Recovery of the cone $b$-wave after a bright orange $(\lambda>530 \mathrm{~nm}$; OG-530 colored glass filter) conditioning flash (OCF) estimated to isomerize $1 \%$ of the M-cone pigment. Numbers near the traces are the interflash intervals in milliseconds; the trace labeled $O C F$ is the response to the conditioning flash alone (no probe flash). Right-hand traces show responses to a $\lambda_{\text {nom }}=500 \mathrm{~nm}$ probe flash producing 71,000 photons $\mu \mathrm{m}^{-2}$ at the cornea. Left-hand traces show responses to a broadband UV probe flash (330-390 nm; Schott UG1 glass) producing 43,000 "equivalent" $350 \mathrm{~nm}$ photons $\mu \mathrm{m}^{-2}$ at the cornea; the equivalency is computed with respect to the UV pigment template shown in Figure 6 (Eq. 3). The probe flashes were the most intense the apparatus could generate (Fig. 5A, abscissa). (To eliminate most of the rod signal but keep the cones maximally sensitive, the entire experiment was performed in the presence of a steady $520 \mathrm{~nm}$ background producing $\varphi=750$ photoisomerizations/ sec per rod. To insure absence of any UV light in the orange conditioning flash, the intensity of the flash generated by the same flash unit with a combination of Schott glass filters (UG1 + OG-530 + BG39) was measured with a photodiode; the BG39 filter was added to block infrared light. The photodiode failed to record any signal. This indicates that the OCF produced $<200$ photons $\mu \mathrm{m}^{-2}$ at the cornea in the UV region of the spectrum.)

the cone-driven b-wave; thus, in Figure $6 B$ we approximated the M-cone spectrum below $\sim 450 \mathrm{~nm}$ with the rod spectrum. Judging from this approximation, such contribution is unlikely to exceed $10 \%$ in the spectral region below $\sim 380 \mathrm{~nm}$.

\section{UV-sensitive cone pathway can be suppressed by long-wavelength illumination}

The $\lambda_{\max }$ values of the pigments underlying the mouse conedriven $b$-waves are separated by $\sim 150 \mathrm{~nm}$. We expected that such wide spectral separation would allow isolation of UV-cone-driven signals by selective chromatic adaptation, i.e., isolation in experiments in which M-cone signals were transiently suppressed with an appropriately intense long-wavelength conditioning flash. Figure 7 shows the results of such an experiment, designed as follows. A steady $520 \mathrm{~nm}$ background was applied to suppress most of the rod activity. Then, an intense orange conditioning flash $(\lambda>530$ $\mathrm{nm}$ ) producing $\sim 1 \%$ isomerization of the rhodopsin, and a similar fractional isomerization of the M-cone cone pigment, was

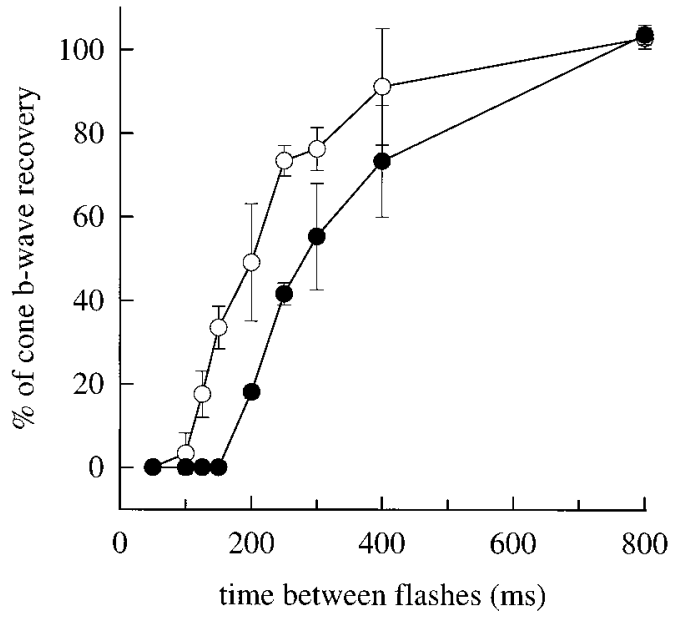

Figure 8. Recovery time course for cone b-wave responses probed with 350 and $500 \mathrm{~nm}$ flashes. Recovery time course for the peak amplitude of the response to a UV probe flash $(\bigcirc)$ and $500 \mathrm{~nm}$ probe flash $(\bullet)$, after exposure to an intense orange conditioning flash at $t=0$. Data points are mean \pm SD for three experiments identical to (and including) that of Figure 7, performed on different mice. The response amplitudes are normalized by the amplitude at $800 \mathrm{msec}$.

delivered $2 \mathrm{sec}$ after the background onset to suppress M-cone signals. A relatively intense probe flash, either broadband UV $(330-390 \mathrm{~nm})$ or $\lambda_{\text {nom }}=500 \mathrm{~nm}$, was delivered at various times from 100 to $800 \mathrm{msec}$ after the orange conditioning flash to monitor sensitivity of signal pathways driven by the UV- and M-cones, respectively. As expected, responsivity to the $500 \mathrm{~nm}$ probe flash was temporarily suppressed by the conditioning flash: the response reappeared at $200 \mathrm{msec}$ and reached $50 \%$ recovery in 350-400 msec. However, quite surprisingly, responsivity to the UV probe flash was also completely suppressed, appearing only after 125 msec. Given the intensity of the probe flashes (Fig. 7, legend), it is clear that the loss of responsivity to both probes is attributable to signal saturation caused by the orange conditioning flash and not mere loss of sensitivity.

In Figure 8, the time courses of recovery of the responses to the $\mathrm{UV}$ and $500 \mathrm{~nm}$ probe flashes are summarized for three animals. In each animal, the orange conditioning flash always completely suppressed responsivity to the UV stimulus, although the recovery of responsivity to the UV probe flash was always more rapid than to the $500 \mathrm{~nm}$ flash. A calculation based on Equation 3 demonstrates that the orange conditioning flash used in the experiment of Figures 7-8 should not produce a number of photoisomerizations of the UV pigment sufficient even to generate a detectable response from the UV-sensitive pathway, much less drive this pathway into saturation. Thus, assuming that the absorbance of the UV pigment at long wavelengths is described by Lamb's (1995) template 2 as fitted to the data in Figure $6 B$, we calculated with Equation 3 that the total light transmitted through the Schott OG530 filter is equivalent to $<200$ quanta $\mu \mathrm{m}^{-2}$ of $350 \mathrm{~nm}$ light at the cornea. On the basis of the absolute sensitivity of the cone-driven b-wave at $350 \mathrm{~nm}, \sim 8 \mathrm{nV}\left(\text { photon } \mu \mathrm{m}^{-2}\right)^{-1}$ (Fig. 5A, Table 1), this amount of $350 \mathrm{~nm}$ light is predicted to elicit a cone b-wave of $<2 \mu \mathrm{V}$; such a response would be practically undetectable and is certainly far from saturation.

\section{Evidence for postreceptor functional distinctiveness of} UV- and M-cone signaling pathways

The experiments illustrated in Figures 7 and 8 cast doubt on the use of any selective chromatic adaptation protocol for completely 

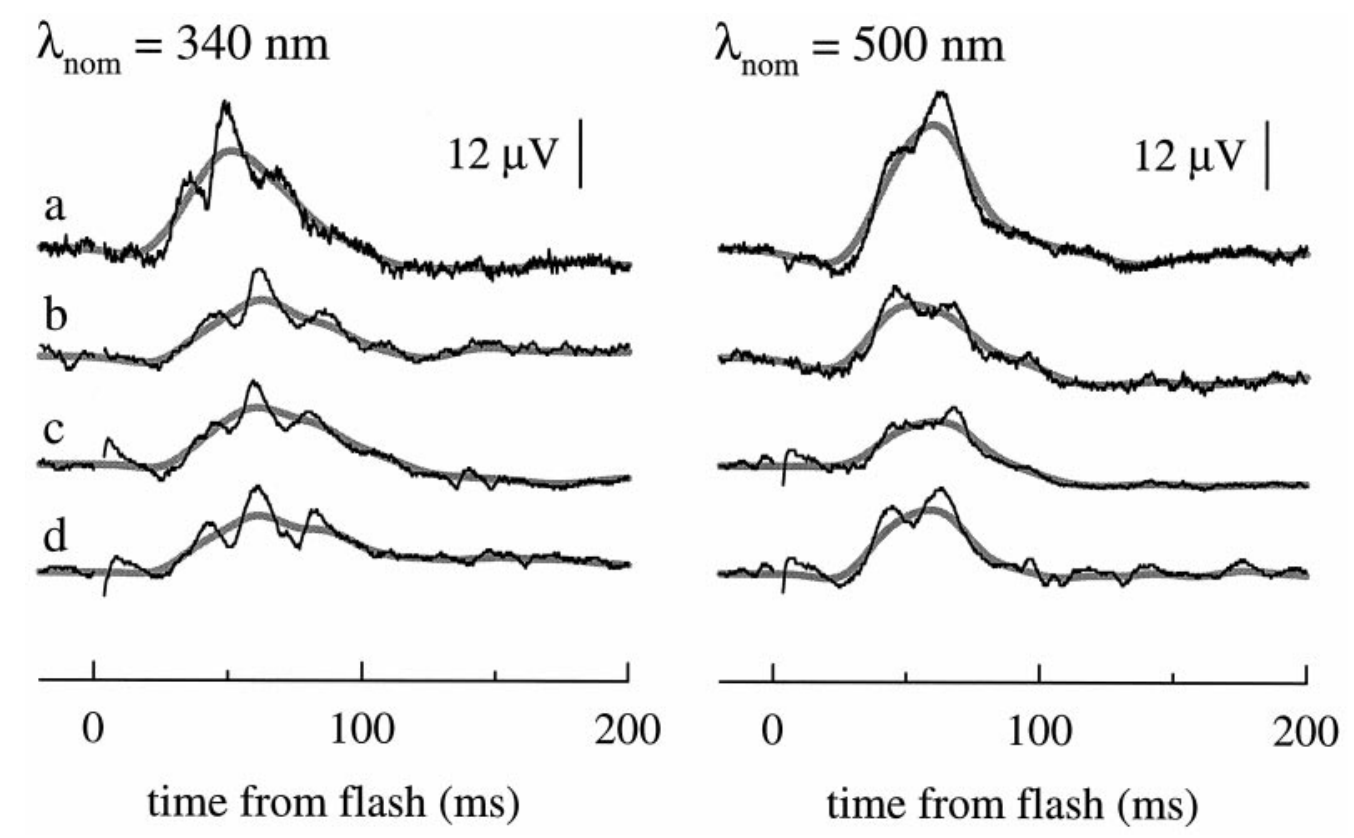

Figure 9. Dim-flash cone-driven b-waves for UV and midwave light flashes. Noisy black traces are the responses of four different mice (a-d) under cone-isolation conditions to $340 \mathrm{~nm}$ (left) and $500 \mathrm{~nm}$ (right) flashes that produce responses of amplitude $<20 \%$ of the maximum amplitude of the cone-driven b-wave; each trace is the average of three to five individual records. Smooth, thicker gray traces were obtained by filtering the responses with a Gaussian filter of bandwidth $16.6 \mathrm{~Hz}$, as in Figure 4.

separating in the ERG signals originating in the UV- and M-cones. Nonetheless, there is reason to believe that the signaling pathways of the two cone classes can be isolated by appropriate monochromatic flashes at the lowest flash strengths that elicit cone-driven responses. The action spectrum data of Figure $6 B$ provide evidence that in the UV region $\mathrm{M}$-cone-driven responses should be negligible near threshold. Additional evidence for isolation near threshold is provided in Figure 4, where it can be seen that the lowest amplitude responses to the 340 and $500 \mathrm{~nm}$ flashes produce responses of different shape: the response to the $340 \mathrm{~nm}$ flash has two to three oscillatory bumps, whereas that to the 520 nm flash exhibits only a single peak. The oscillatory potentials of the lowest amplitude traces in Figure 4 may have been artificially smoothed by extensive on-line averaging. To provide further evidence about the shapes of the dim-flash responses in Figure 9 we present responses from four additional animals. A pattern emerges: the dim-flash, UV-driven responses characteristically exhibit a triple oscillation, with the middle oscillatory peak coinciding with the peak of the smoothed response. In contrast, the dim-flash responses to $500 \mathrm{~nm}$ light typically exhibit only a pair of oscillatory peaks, peaks that are not centered on the smoothed response maximum and have more variation in relative amplitude across animals than the UV-driven oscillations.

\section{DISCUSSION}

\section{Murine cone a-wave}

On the basis of its rise time, its relative insensitivity to steady light, and its magnitude, we tentatively identify the cornealnegative ERG component measured in the presence of rodsaturating backgrounds (Fig. $2 A, C$ ) as a cone a-wave, i.e., a field potential caused by suppression of cone circulating current. The presumed saturated amplitude of the murine cone a-wave was found to be $\sim 20 \mu \mathrm{V}$, roughly consistent with expectation. Specifically, based on a cone $/$ rod density ratio $\rho_{\text {cone }} / \rho_{\text {rod }}=0.03$
(Carter-Dawson and LaVail, 1979a), $a_{\text {max,rod }}=360 \pm 132 \mu \mathrm{V}$ (Table 1), and the assumption that cones and rods have equal circulating currents, the rod gauge (Eq. 18) predicts the saturating cone a-wave to be $0.03 \times 360 \mu \mathrm{V}=12 \mu \mathrm{V}$.

\section{Rod and cone spectral sensitivities}

The spectral sensitivities of murine rods and cones (Fig. 6) derived from our ERG measurements are consistent with known facts about murine photopigments (see introductory remarks). An interesting quantitative feature of the murine rod spectrum in situ (Fig. 6A) is its relatively high sensitivity in the $\beta$-band (UV) spectral region, a sensitivity that ranges from 30 to $40 \%$ of that at the $\lambda_{\text {max }}$; from this it is clear that mice have effective rod vision in the UV region of the spectrum.

\section{Absolute magnitudes of rod- and cone-driven b-waves: implications for cell densities}

Substantial evidence has now accumulated that the mammalian scotopic b-wave is generated primarily by glutamate-modulated currents of rod bipolar cells (e.g., see Robson and Frishman, 1995, 1996). The rod b-wave maximum in mice under our recording conditions is $b_{\text {max,rod }} \approx 400 \mu \mathrm{V}$, whether this number is obtained by direct analysis of the saturation function of the scotopic b-wave (Pennesi et al., 1998; Pugh et al., 1998) or by subtraction of the cone $b_{\max }$ from the total rod + cone b-wave amplitude $b_{\text {max,rod }+ \text { cone }}$ (Table 1). Assuming a rod/rod-bipolar (RB) ratio $\rho_{\mathrm{RB}} / \rho_{\text {rods }}=1: 20$ to 1:50 (Dacheux and Raviola, 1986; Freed et al., 1987), the rod gauge (Eq. 18) predicts the maximum rod bipolar "generator current" to be $J_{\mathrm{RB}}=500-1250 \mathrm{pA}$.

There are many similarities between rod bipolars and cone on-bipolar cells-including their radial retinal location and arborization and their likely use of G-protein signaling cascades coupled to mGluR6 metabotropic receptors (Nakajima et al., 1993; Vardi et al., 1993). Thus, on the assumption that the 
cone-driven b-wave originates in the signal generator current of cone on-bipolars (COB), based on the relative amplitudes of the rod b-wave maximum $(\approx 400 \mu \mathrm{V})$ and cone-driven b-wave maximum $(\approx 200 \mu \mathrm{V})$ (Table 1), Equation 18 generates the prediction $\rho_{\mathrm{RB}} \approx 2 \rho_{\mathrm{COB}} J_{\mathrm{COB}}$. If the cone on-bipolar and rod bipolar have the same magnitude maximum generator currents $\left(J_{\mathrm{RB}} \approx J_{\mathrm{COB}}\right)$, then the spatial density $\rho_{\mathrm{COB}}$ of cone on-bipolars is predicted to be one-third that of the bipolars, $\rho_{\mathrm{RB}}$, in mice.

\section{Absolute sensitivities of rod- and cone-driven b-waves}

The absolute sensitivity of the murine rod b-wave at the cornea is 50-100 $\mu \mathrm{V}$ (photon $\left.\mu \mathrm{m}^{-2}\right)^{-1}$; this sensitivity can be derived from scotopic b-wave response versus intensity data of several investigations, as summarized elsewhere (Pennesi et al., 1998; Pugh et al., 1998). In contrast to that of the rod-driven b-wave, the absolute sensitivity of the UV-cone-driven b-wave at the cornea is $\sim 8 \mathrm{nV}$ (photon $\left.\mu \mathrm{m}^{-2}\right)^{-1}, \sim 10,000$-fold lower than that of the rod b-wave. How might such an enormous difference in sensitivity arise? At least three factors are at play: (1) the difference in individual rod- and cone-collecting areas "at the cornea," (2) the intrinsic differences in sensitivities of rods and cones, and (3) the difference in photoreceptor $\rightarrow$ bipolar convergence.

The first factor arises in the physical optics of rods and cones: the cone-collecting area at the cornea is reduced by the StilesCrawford (SC) effect (Stiles and Crawford, 1933), whereby the waveguiding properties of cones diminish the capture of light not impinging axially on the cone inner segments (Snyder and Pask, 1973). Because the mouse eye with fully dilated pupil has a large numerical aperture (Remtulla and Hallett, 1985), light from the pupil margin can strike the retina at angles as great as $30^{\circ}$. Assuming parameters describing human cones (Snyder and Pask, 1973), we estimate that the SC effect could reduce the effective area of the dilated mouse pupil for cone-driven signals from its physical area of $3.1 \mathrm{~mm}^{2}$ to approximately one-third this value.

A second and major factor involved in the insensitivity of cone b-waves relative to those of rods is the absolute sensitivities of the photoreceptors themselves. From intracellular recordings, Schneeweis and Schnapf (1995) estimated the absolute sensitivities of the dim-flash response peaks of primate rods and cones to be $\sim 1 \mathrm{mV} / \Phi$ and $5 \mu \mathrm{V} / \Phi$, respectively, giving a likely output gain ratio of 200 .

A third factor reducing the light sensitivity at the cornea of cone-driven b-wave responses relative to those of rods is the photoreceptor $\rightarrow$ bipolar convergence ratios. The convergence ratio for rods $\rightarrow$ RBs is likely 20-30 times greater than that for cones $\rightarrow$ cone on-bipolars (Dacheux and Raviola, 1986; Freed et al., 1987). This convergence factor can be thought of in terms of the total surface area at the retina available for collecting photons, which is $\sim 20-30$ times greater for rods than cones.

In summary, the product of the three factors considered, $3 \times$ $200 \times 20=12,000$, appears more than sufficient to account for the 10,000 -fold ratio of rod- to cone-driven b-waves at the cornea. Because the estimates of the individual factors must be considered rough, however, differential "synaptic gain" between photoreceptors and bipolars cannot be ruled out.

\section{The nature of the interaction of signals originating in murine UV- and $\mathrm{M}$-cone photopigments}

The most surprising finding of this investigation is reported in Figures 7 and 8: a long-wave conditioning flash can completely suppress responsitivity of the UV-cone-driven signaling pathway. (The cone-signal interaction hypothesis discussed in this section underscores a weakness in current cone nomenclature. In keeping with past usage, we will continued to identify cones that express primarily the UV pigment as "UV-cones" and cones that express primarily the M pigment as "M-cones.") Given that photons captured by the UV photopigment cannot be the cause, what might underlie the complete loss of responsivity to UV flashes seen in Figures 7-8? Clearly, photons captured by a visual pigment with absorption in the spectral band of the orange conditioning flash must be forcing the pathway for UV-cone-driven responses into saturation, at or before the level of the UVsensitive b-wave generator. It follows that photons captured by the M-cone photopigment must be the cause of the loss of sensitivity to UV flashes, and thus that the M pigment and UV pigment signaling pathways must be linked. One hypothesis for the loss of UV responsivity is that M-cone signals block all UV-cone signals at some site of convergence at or beyond the cone $\rightarrow$ bipolar synapse. Because M-cones are located exclusively in the dorsal half of the mouse retina and most of the UV-cones are in the ventral half (Szel et al., 1992), it is unlikely that the signals from M-cones could completely suppress UV-cone signaling at any site of convergence in the retina. An alternative hypothesis that can explain the results in Figures 7-8 is that the $\mathrm{M}$-cone pigment is expressed to some degree in all the UV-cones. This latter hypothesis is not without precedent: in suction electrode recordings from isolated salamander cones, Makino and Dodd (1996) found that cones with peak sensitivity in the UV have action spectra reflecting expression of three distinct opsins. Some evidence for the coexpression of the $\mathrm{M}$ pigment in the UV-cones of mice was provided by the immunocytochemical investigation of Rohlich et al. (1994), who reported that in the transitional zone of the retina between dorsal and ventral retina, there are many cones labeled by antibodies to both S- (UV) and M-cone pigments. Even more pertinent to our results are the very recent results of Gloesman and Ahnelt (1998), who have found with antibodies to the UV- and M-cone pigments double-labeling of all but a small minority of UV-labeled cones in the ventral retina.

On the basis of the hypothesis that the cones expressing primarily the UV pigment also express some $\mathrm{M}$ pigment, our data yield a rough upper limit to the amount of $\mathbf{M}$ pigment present, as follows. The time course of recovery of the response to the $500 \mathrm{~nm}$ flash is seen in Figure 8 to be shifted by $\sim 90 \mathrm{msec}$ to the right of the recovery of the response to the UV flash (consider, for example, the lateral shift between the curves at the times of $20 \%$ recovery). Given that the "dominant time constant" $\tau_{\mathrm{c}}$ of mammalian cone recovery from saturating flashes has been estimated to be $\sim 25 \mathrm{msec}$ (T. Kraft, personal communication), and the assumption that the b-wave recovery tracks the cone recovery, the leftward shift of $\Delta T=90$ msec of the recovery to the UV flash dictates that the UVcones contain no more than $\exp \left(-\Delta T / \tau_{\mathrm{c}}\right)=0.03$ of the M-cone pigment [for analysis of the concept of a dominant recovery time constant, see Pepperberg et al. (1996) and Nikonov et al. (1998)]. In closing we note that coexpression of even $3 \%$ of $\mathrm{M}$ pigment in the UV-cones provides a satisfactory explanation of the ability of intense white flashes from flash units or apparatuses that filter out UV light to saturate murine conedriven b-wave signals.

\section{REFERENCES}

Alpern M, Fulton AB, Baker BN (1987) "Self-screening" of rhodopsin in rod outer segments. Vision Res 27:1459-1470. 
Baylor DA, Nunn BJ, Schnapf JL (1984) The photocurrent, noise and spectral sensitivity of rods of the monkey Macaca fascicularis. J Physiol (Lond) 357:575-607.

Breton ME, Schueller AW, Lamb TD, Pugh Jr EN (1994) Analysis of ERG a-wave amplification and kinetics in terms of the G-protein cascade of phototransduction. Invest Ophthalmol Vis Sci 35:295-309.

Calderone JB, Jacobs GH (1995) Regional variations in the relative sensitivity to UV light in the mouse retina. Vis Neurosci 12:463-468.

Carter-Dawson LD, LaVail MM (1979a) Rods and cones in the mouse retina. I. Structural analysis using light and electron microscopy. J Comp Neurol 188:245-262.

Carter-Dawson LD, LaVail MM (1979b) Rods and cones in the mouse retina. II. Autoradiographic analysis of cell generation using tritiated thymidine. J Comp Neurol 188:263-272.

Chiu MI, Zack DJ, Wang Y, Nathans J (1994) Murine and bovine blue cone pigment genes: cloning and characterization of two new members of the S family of visual pigments. Genomics 21:440-443.

Cideciyan AV, Jacobson SG (1996) An alternative phototransduction model for human rod and cone ERG a-waves: normal parameters and variation with age. Vision Res 36:2609-2621.

Cobbs WH, Pugh Jr EN (1987) Kinetics and components of the flash photocurrent of isolated retinal rods of the larval salamander, Ambystoma tigrinum. J Physiol (Lond) 394:529-572.

Dacheux RF, Raviola E (1986) The rod pathway in the rabbit retina: a depolarizing bipolar and amacrine cell. J Neurosci 6:331-345.

Dartnall HJA (1953) The interpretation of spectral sensitivity curves. $\mathrm{Br}$ Med Bull 9:24-30.

Deegan 2d JF, Jacobs GH (1993) On the identity of the cone types of the rat retina. Exp Eye Res 56:375-377.

Freed MA, Smith RG, Sterling P (1987) Rod bipolar array in the cat retina: pattern of input from rods and GABA-accumulating amacrine cells. J Comp Neurol 266:445-455.

Fulton AB, Rushton WAH (1978) The human rod ERG: correlation with psychophysical responses in light and dark adaptation. Vision Res 18:793-800.

Gloesman M, Ahnelt PK (1998) Co-expression of M-opsin and S-opsin extends over the entire inferior mouse retina. Invest Ophthalmol Vis Sci 39:S1059.

Hagins WA, Penn RD, Yoshikami S (1970) Dark current and photocurrent in retinal rods. Biophys J 10:380-412.

Heynen H, Wachmeister L, van Norren D (1985) Origin of oscillatory potentials in primate retina. Vision Res 25:1365-1373.

Hood DC, Birch DG (1993) Human cone receptor activity: the leading edge of the a-wave and models of receptor activity. Vis Neurosci 10:857-871.

Hood DC, Birch DG (1995) Phototransduction in human cones measured using the a-wave of the ERG. Vision Res 35:2801-2810.

Jacobs GH, Neitz J, Deegan 2d JF (1991) Retinal receptors in rodents maximally sensitive to ultraviolet light. Nature 353:655-656.

Kraft TW, Schneeweis DM, Schnapf JL (1993) Visual transduction in human rod photoreceptors. J Physiol (Lond) 464:747-765.

Lamb TD (1995) Photoreceptor spectral sensitivities: common shape in the long-wavelength region. Vision Res 35:3083-3091.

Lamb TD, Pugh Jr EN (1992) A quantitative account of the activation steps involved in phototransduction in amphibian photoreceptors. J Physiol (Lond) 449:719-757.

Lem J, Makino CL (1996) Phototransduction in transgenic mice. Curr Opin Neurobiol 6:453-458.

Lyubarsky AL, Pugh Jr EN (1996) Recovery phase of the murine rod photoresponse reconstructed from electroretinographic recordings. J Neurosci 16:563-571.

Makino CL, Dodd RL (1996) Multiple visual pigments in a photoreceptor of the salamander retina. J Gen Physiol 108:27-34.
Mansfield RJW (1985) Primate photopigments and cone mechanisms. In: The visual system: (Fein A, Levine J, eds), pp 89-106. New York: Alan Liss.

Nakajima Y, Iwakabe H, Akazawa C, Nawa H, Shigemoto R, Mizuno N, Nakanishi S (1993) Molecular characterization of a novel retinal metabotrophic glutamate receptor mGluR6 with a high agonist selectivity for L-2-amino-4-phosphonobutyrate. J Biol Chem 268:11868-11873.

Nakatani K, Tamura T, Yau KW (1991) Light adaptation in retinal rods of the rabbit and two other nonprimate mammals. J Gen Physiol 97:413-435

Nikonov S, Engheta, N, Pugh Jr EN (1998) Kinetics of the dark-adapted salamander rod photoresponse. J Gen Physiol 111:7-37.

Peachey NS, Alexander KR, Fishman GA (1987) Rod and cone system contributions to oscillatory potentials: an explanation for the conditioning flash effect. Vision Res 27:859-866.

Peachey NS, Goto Y, Al-Ubaidi MR, Naash MI (1993) Properties of the mouse cone-mediated electroretinogram during light adaptation. Neurosci Lett 162:9-11.

Penn RD, Hagins WA (1972) Kinetics of the photocurrent of retinal rods. Biophys J 10:380-412.

Pennesi ME, Lyubarsky AL, Pugh Jr EN (1998) Extreme responsiveness of the pupil of the dark adapted mouse to steady retinal illumination. Invest Ophthalmol Vis Sci 11:2148-2156.

Pepperberg DR, Birch DG, Hofman KP, Hood DC (1996) Recovery kinetics of human rod phototransduction inferred from the two branched alpha-wave saturation function. J Opt Soc Am A 13:586-600.

Pugh Jr EN, Falsini B, Lyubarsky AL (1998) The origin of the major rod- and cone-driven components of the rodent electroretinogram, and the effect of age and light-rearing history on the magnitudes of these components. In: Photostasis and related topics (Williams TP, Thistle AB, eds), pp 93-128. New York: Plenum.

Reiser M, Williams TP, Pugh Jr EN (1996) The effect of light history on the aspartate-isolated fast-PIII response of the albino rat retina. Invest Ophthalmol Vis Sci 37:321-229.

Remtulla S, Hallett PE (1985) A schematic eye for the mouse, and comparisons with the rat. Vision Res 25:21-31.

Robson JG, Frishman LJ (1995) Response linearity and kinetics of the cat retina: the bipolar cell component of the dark-adapted electoretinogram. Vis Neurosci 12:837-850.

Robson JG, Frishman LJ (1996) Photoreceptor and bipolar-cell contributions to the cat electroretinogram: a kinetic model for the early part of the flash response. J Opt Soc Am 13:613-622.

Rohlich P, van Veen T, Szel A (1994) Two different visual pigments in one retinal cone cell. Neuron 13:1159-1166.

Schneeweis DM, Schnapf JL (1995) Photovoltage of rods and cones in the macaque retina. Science 268:1053-1056.

Smith NP, Lamb TD (1997) The a-wave of the human electroretinogram recorded with a minimally invasive technique. Vision Res 37: 2943-2952.

Snyder AW, Pask C (1973) The Stiles-Crawford effect: explanation and consequences. Vision Res 13:1115-1137.

Stiles WS, Crawford BH (1933) The luminous efficiency of rays entering the pupil at different points. Proc R Soc Lond B Biol Sci 112:428-450.

Sun H, Macke JP, Nathans J (1997) Mechanisms of spectral tuning in the mouse green cone pigment. Proc Natl Acad Sci USA 94:8860-8865.

Szel A, Rohlich P, Caffe AR, Juliusson B, Aguirre G, van Veen T (1992) Unique topographic separation of two spectral classes of cones in the mouse retina. J Comp Neurol 325:327-342.

Vardi N, Matesic DF, Manning DR, Liebman PA, Sterling P (1993) Identification of a G-protein in depolarizing rod bipolar cells. Vis Neurosci 10:473-478.

Wyszecki G, Stiles WS (1983) Color science: concepts and methods, quantitative data and formulae, Ed 2. New York: Wiley. 